\title{
Identical Twins as a Facial Similarity Benchmark for Human Facial Recognition
}

John Andrew McCauley

West Virginia University, jamccauley@mix.wvu.edu

Follow this and additional works at: https://researchrepository.wvu.edu/etd

Part of the Other Electrical and Computer Engineering Commons

\section{Recommended Citation}

McCauley, John Andrew, "Identical Twins as a Facial Similarity Benchmark for Human Facial Recognition" (2021). Graduate Theses, Dissertations, and Problem Reports. 10286.

https://researchrepository.wvu.edu/etd/10286

This Thesis is protected by copyright and/or related rights. It has been brought to you by the The Research Repository @ WVU with permission from the rights-holder(s). You are free to use this Thesis in any way that is permitted by the copyright and related rights legislation that applies to your use. For other uses you must obtain permission from the rights-holder(s) directly, unless additional rights are indicated by a Creative Commons license in the record and/ or on the work itself. This Thesis has been accepted for inclusion in WVU Graduate Theses, Dissertations, and Problem Reports collection by an authorized administrator of The Research Repository @ WVU. For more information, please contact researchrepository@mail.wvu.edu. 


\title{
Identical Twins as a Facial Similarity Benchmark for Human Facial Recognition
}

\author{
John McCauley
}

Thesis submitted

to the Benjamin M. Statler College of Engineering and Mineral Resources at West Virginia University

in partial fulfilment of the requirements for the degree of

Master of Science in

Electrical Engineering

\author{
Jeremy Dawson, Ph.D., Chair \\ Nasser Nasrabadi, Ph.D. \\ Matthew Valenti, Ph.D.
}

Lane Department of Computer Science and Electrical Engineering Morgantown, WV

2021

Keywords: Facial Recognition, Facial Similarity, Identical Twins, Deep Learning Copyright (C) 2021 John McCauley 


\begin{abstract}
Identical Twins as a Facial Similarity Benchmark for Human Facial Recognition
\end{abstract}

John McCauley

The problem of distinguishing identical twins and non-twin look-alikes in automated facial recognition (FR) applications has become increasingly important with the widespread adoption of facial biometrics. Due to the high facial similarity of both identical twins and look-alikes, these face pairs represent the hardest cases presented to facial recognition tools. The effect of these highly similar face pairs on these tools is important to investigate to ensure that facial recognition tools can adequately address these problems. Additionally, analyzing the facial similarity of these face pairs allows for a better understanding of the differences between the comparison score returned by a facial recognition tool, and a similarity score based on the perceived facial similarity of the faces in question. This analysis allows for an investigation into the role that facial similarity plays in the determination of face comparison scores in any face recognition approach.

This work presents an application of one of the largest twin datasets compiled to date to address two FR challenges: 1) determining a baseline measure of facial similarity between identical twins and 2) applying this similarity measure to determine the impact of doppelgangers, or look-alikes, on FR performance for large face datasets. The facial similarity measure is determined via a deep convolutional neural network. This network is trained on a tailored verification task designed to encourage the network to group together highly similar face pairs in the embedding space and achieves a test AUC of 0.9799. The proposed network provides a quantitative similarity score for any two given faces and has been applied to large-scale face datasets to identify similar face pairs. An additional analysis which correlates the comparison score returned by a facial recognition tool and the similarity score returned by the proposed network has also been performed. 


\section{Acknowledgements}

I would first like to give my deepest thanks to my academic and research advisor Dr. Jeremy Dawson. His mentorship throughout my time as a graduate research assistant has been invaluable to the completion of this work, as well as my growth as an engineer and researcher. I also extend my gratitude to my committee members Dr. Nasser Nasrabadi and Dr. Matthew Valenti. I thank them both for their interest in my work and for lending their expertise in the fields of neural networks and biometrics.

I am also grateful to my colleagues and lab mates at the WVU biometrics lab for their friendship and assistance in collecting the data used in this work. A special thank you to Sobhan Soleymani for providing guidance in the development of neural networks.

Finally, I must give thanks to my parents, brothers, and the many friends and family members who have given me encouragement throughout my years of study. Without their support, this accomplishment would not have been possible. Thank you. 


\section{Table of Contents}

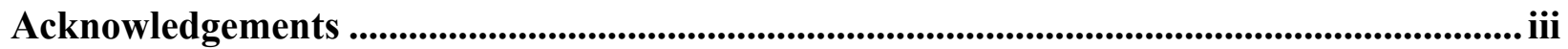

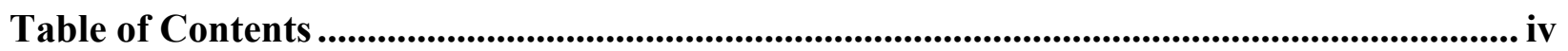

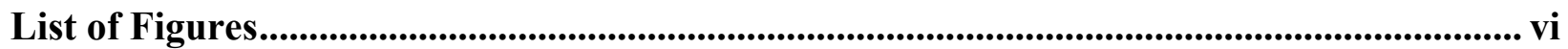

List of Tables .........................................................................................................................................ii

List of Equations ........................................................................................................... ix

Chapter 1: Introduction .......................................................................................................................... 1

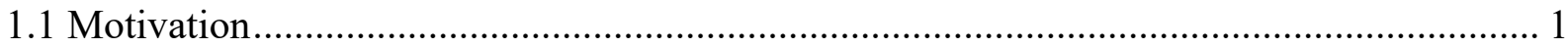

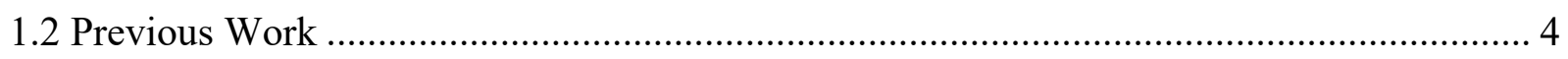

1.2.1 Biometric Identification of Identical Twins ............................................................ 4

1.2.2 Facial Recognition of Identical Twins .................................................................... 5

1.2.3 Facial Recognition of Look-alikes........................................................................... 7

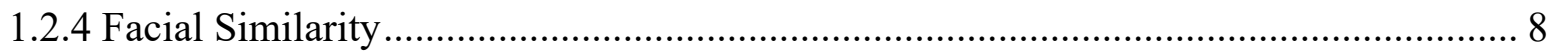

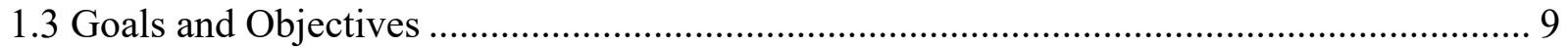

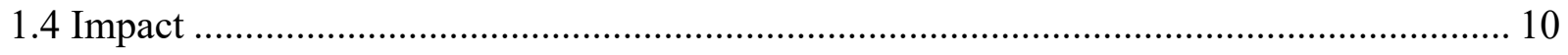

Chapter 2: Theory and Background ................................................................................................ 12

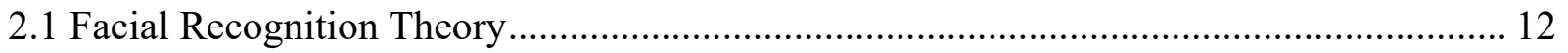

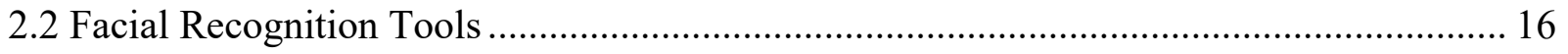

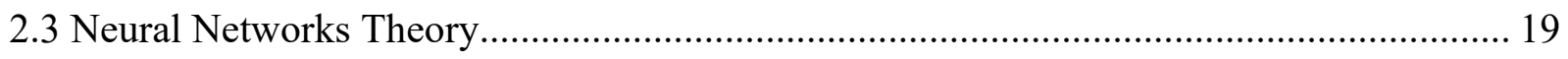

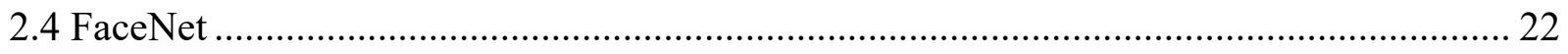

Chapter 3: Datasets, Matching Experiments, and Similarity Network..................................... 28

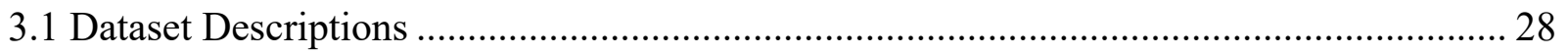

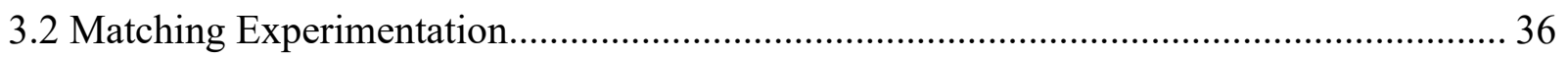

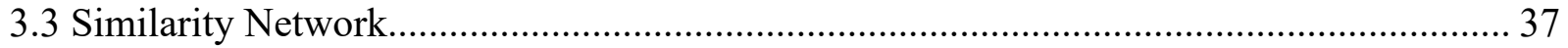


Chapter 4: Results............................................................................................................................ 41

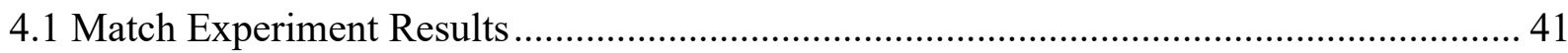

4.1.1 Identical Twin Baseline Experiments .......................................................................... 41

4.1.2 All-to-all Non-mated Matching Experiments …………………………………....... 43

4.1.3 Twin Dataset Match Experiment Results ................................................................... 44

4.1.4 Non-twin Dataset Match Experiment Results.......................................................... 45

4.1.5 Large Scale Non-Twin Dataset Match Experiment Results ........................................ 47

4.1.6 Match Scores Above Experimental Threshold $T$...................................................... 48

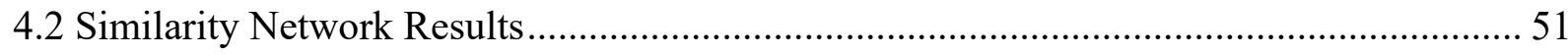

4.2.1 Network Training and Testing ......................................................................... 51

4.2.2 Identical Twin Similarity Baseline ............................................................................ 52

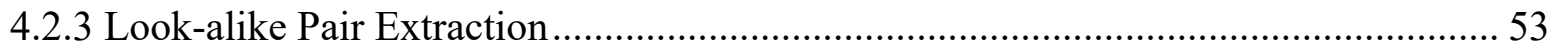

4.2.4 Comparison Score Versus Similarity Score Analysis................................................ 54

4.2.5 Frequency of Look-alike Identity Occurrence Estimation.............................................5 58

Chapter 5: Conclusions .................................................................................................................................. 61

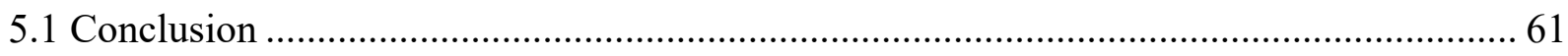

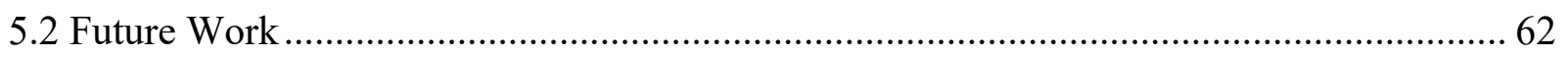

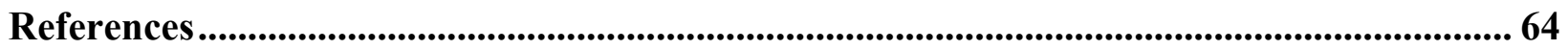




\section{List of Figures}

Figure 1: An example of an identical twin pair. ............................................................ 2

Figure 2: Celebrity look-alikes, Will Ferrell and Chad Smith............................................... 2

Figure 3: Example of comparison decision threshold [21] ................................................. 14

Figure 4: An example convolutional neural network architecture [30]................................ 21

Figure 5: Visual representation of max-pooling step [31] ................................................ 21

Figure 6: Visual representation of the triplet loss function [26] ......................................... 24

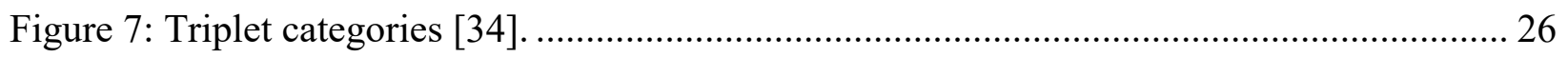

Figure 8: Twin dataset gender and ethnicity demographics chart. ........................................ 30

Figure 9: Twin dataset ethnicity demographics chart. .................................................. 31

Figure 10: Twin dataset age group demographics chart. ................................................. 32

Figure 11: Non-twin dataset gender and ethnicity demographics chart. ................................. 33

Figure 12: Non-twin dataset ethnicity demographics chart. .................................................. 34

Figure 13: Non-twin dataset age group demographics chart. ............................................ 35

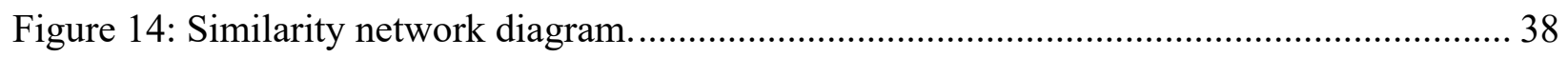

Figure 15: Twin baseline match experiment results, Neurotechnology matcher....................... 42

Figure 16: Twin baseline match experiment results, FaceNet matcher. .................................. 43

Figure 17: Twin dataset all-to-all non-mated match experiment results, Neurotechnology COTS

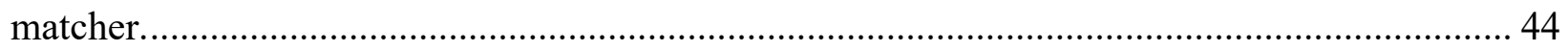

Figure 18: Twin dataset all-to-all non-mated match experiment results, FaceNet matcher........ 45

Figure 19: Non-twin dataset all-to-all non-mated match experiment results, Neurotechnology

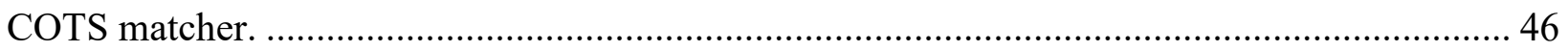

Figure 20: Non-twin dataset all-to-all non-mated match experiment results, FaceNet matcher. . 47

Figure 21: Large Scale non-twin dataset all-to-all non-mated match experiment results, FaceNet

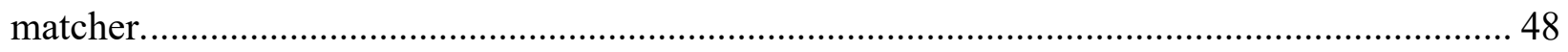

Figure 22: Train ROC curve of similarity network on the tailored verification task................ 51

Figure 23: Test ROC curve of similarity network on the tailored verification task.................. 52

Figure 24: Twin similarity score baseline experiment......................................................... 53 
Figure 25: Examples of highly similar and dissimilar face pairs as determined by the proposed similarity network.

Figure 26: High COTS match score face pairs and the corresponding similarity scores for each pair.

Figure 27: Correlation scatter plot of comparison scores and similarity scores, COTS matcher. 56 Figure 28: Correlation scatter plot of comparison scores and similarity scores, FaceNet matcher.

Figure 29: Number of look-alike identities returned from the large scale non-twin dataset based on similarity threshold used...... 


\section{List of Tables}

Table 1: FaceNet NN2, neural network architecture. ..................................................... 24

Table 2: Twin dataset non-mated match experiment results, comparison scores above

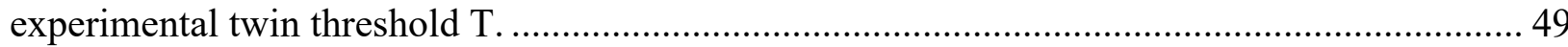

Table 3: Non-twin dataset non-mated match experiment results, comparison scores above

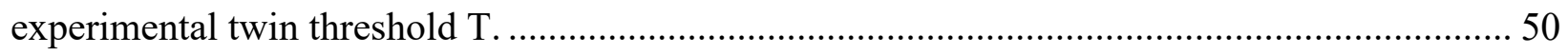

Table 4: Large scale non-twin dataset non-mated match experiment results, comparison scores

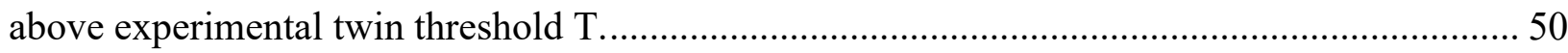




\section{List of Equations}

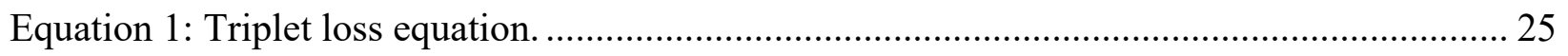

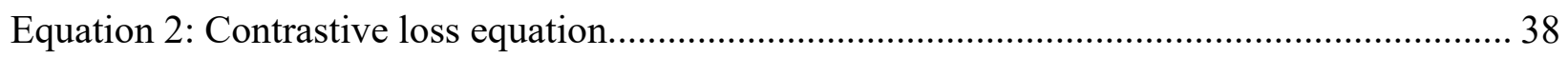




\section{Chapter 1: Introduction}

\subsection{Motivation}

Identical or mono-zygotic twins pose an important and interesting problem to facial recognition (FR) systems. Due to the high level of facial similarity exhibited between identical twin pairs, these individuals are often mis-identified by automatic facial recognition systems, an example identical twin pair is shown in Figure Figure 1. Several studies have shown this to be case, with one study from Paone et al. [1] showing that the average facial recognition system has a significantly higher equal error rate (EER) when presented with a population of identical twins versus a non-twin population, even under ideal imaging conditions. While identical twins present the worst case of facial similarity, this problem is also extended to non-related individuals with high facial similarity, often known as look-alikes or doppelgangers. A well-known celebrity lookalike pair, Will Ferrell and Chad Smith, is shown in Figure Figure 2. A study of look-alike recognition by Sun et al. [2] found that even deep-learning based approaches to facial recognition have a difficult time accurately identifying look-alike pairs. Look-alike impacts on face recognition is especially relevant due to the rapid increase in the size of face datasets currently being seen. As these face datasets increase in size, they include a larger number of distinct identities, and with each added identity, the chance of encountering a look-alike pair within the dataset increases. Each of these problems represent some of the hardest cases presented to facial recognition systems. As such, these problems must be studied and understood to ensure the successful implementation of facial recognition technology in the future. 


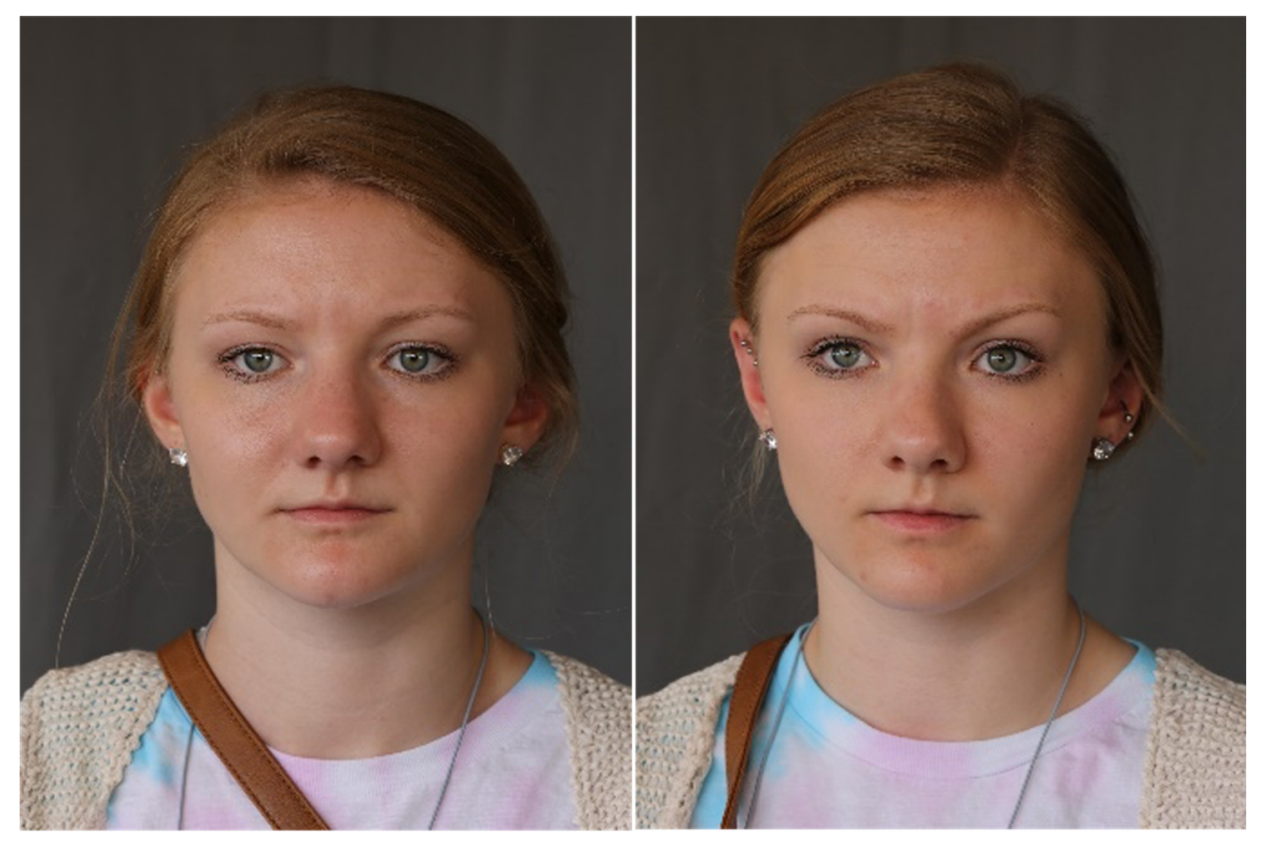

Figure 1: An example of an identical twin pair.

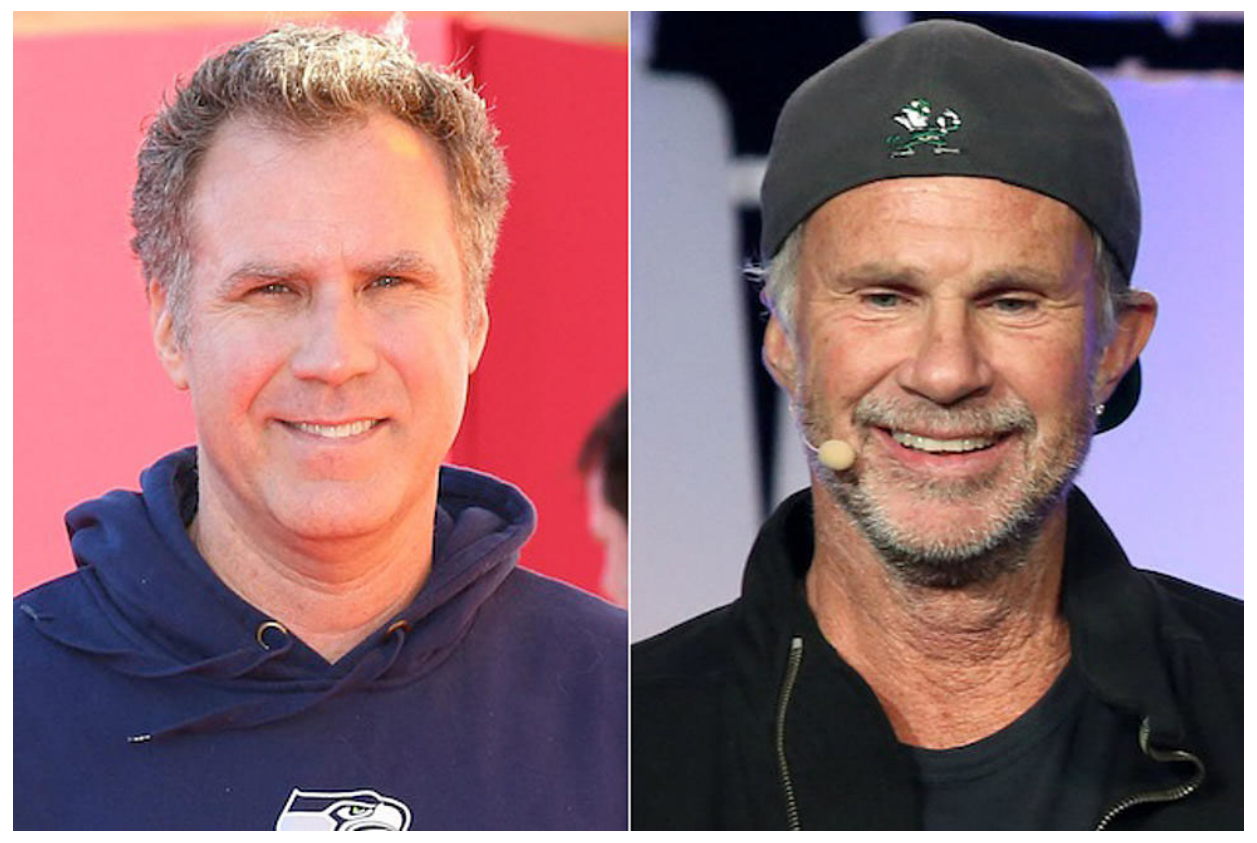

Figure 2: Celebrity look-alikes, Will Ferrell and Chad Smith. 
The challenges caused by identical twins and look-alikes are rooted in the fact that the face comparisons in question happen between identities with high facial similarity. In both cases, two identities are matched against one another in a non-mated comparison in which a comparison or match score is returned for the pair. Due to the high facial similarity of the pair, errors and misidentifications are more likely to occur in these cases. Here, it becomes important to make a distinction between the comparison (or match) score generated by a face matcher and the facial similarity of the identities under comparison. In both cases, face images are being evaluated against one another to return some comparison result. In the case of the comparison score, the intent is to return an accurate identification of the individual(s) in the images in question, whereas the similarity measure seeks to quantify the facial similarity of the identities within the images. This distinction is important, as the comparison score returned by a facial recognition tool may not be directly related or determined by the facial similarity of the individuals. This work seeks to provide greater understanding of the relationship between facial similarity and the comparison score returned by a facial recognition tool.

The research efforts presented in this work are important to facial recognition at large because they establish a worst-case baseline of facial similarity in all face comparisons from face images with known high similarity: those of identical twins. This result represents the most challenging case of non-mated face comparison in general and quantifies the average facial similarity of identical twin pairs. In addition to this baseline measurement, this work also has value in its method of determining facial similarity. This method utilizes one of the largest databases of identical twin images to train a deep convolutional neural network to quantify facial similarity. This measure has further importance in its use in determining highly similar faces in any face dataset. Identifying highly similar faces has several pertinent applications, two of which being the 
selection of appropriately similar faces for morphed face pair generation, and the evaluation of the difficulty of face datasets. Furthermore, this measure of facial similarity can provide key insights into the relationship between facial similarity and the comparison score returned by a facial recognition tool.

\subsection{Previous Work}

\subsubsection{Biometric Identification of Identical Twins}

Biometric identification of identical twins has been studied extensively in many different modalities. In one of the earliest works concerning the biometric identification of identical twins, Jain et al. [3] used the fingerprints of twin pairs to accurately differentiate between the twin individuals. This study employed a dataset of 94 identical twin fingerprints and found that the tested fingerprint recognition tool saw only small performance decreases when presented with identical twins. In a study from Sun et al. [4], multiple modalities of biometric identification were explored. This work examined three biometric modalities, fingerprint, iris, and face. This work found iris to perform the best out of the three modalities, with results for identical twins being just as good as those for non-twin individuals. Facial recognition was the worst performing of the three, showing significantly higher error rate for twins as compared to that of non-twin individuals. In the first study of ear recognition of identical twins [5], Nejati et al. showed that ear recognition techniques can be applied successfully to identical twin datasets. This study developed a new ear recognition algorithm and applied the algorithm to the largest available dataset of identical twins at the time. Their results found recognition accuracy up to $92 \%$ on the twin dataset, confirming that ear recognition is a viable tool for the biometric recognition of twins. Multiple surveys of 
identical twin recognition have confirmed the challenges, and successes, of twin recognition. A survey from Ricanek and Mahalingam [6] discusses the differences seen in identical twins as the twins age. This survey compiles research that indicates identical twins become easier to differentiate as they get older due to age-based changes in their biometric features. The conclusions of this survey indicate that experiments attempting to differentiate between two identical twins will perform better on older twin pairs due to epigenetic changes in the twins' biometrics. In a later survey of the field, Bowyer and Flynn [7] discuss a multitude of biometric recognition techniques used to differentiate twins. They highlight the difficulty of facial recognition of twins through multiple studies, discuss the minor accuracy degradation seen in twin fingerprint recognition as compared to non-twin fingerprint recognition, and show the success of several works exploring iris recognition of identical twins. The survey also discusses the need for larger and more robust datasets of identical twins, as well as the need for further research in speaker recognition and handwriting recognition of identical twins.

\subsubsection{Facial Recognition of Identical Twins}

Facial recognition is quickly becoming one of the most widely used biometric modalities, due to its high acceptance among the general public, ease of data capture, and accuracy. This wide scale implementation makes it more important than ever to evaluate the hardest cases presented to facial recognition systems, such as identical twin pairs. One of the earliest studies on the face recognition of twins employed the Notre Dame/West Virginia University Twins Days dataset, and found that COTS face matchers could identify twins when imaging conditions were ideal (i.e., studio lighting, neutral expression), but performance was measurably decreased when the test images were captured under ambient lighting or had expression variation [8]. A second study 
analyzing the performance of FR algorithms using the Twins Days dataset showed that significant work is still needed to improve the performance of face recognition of identical twins [9]. This study tested the performance of both COTS and academic matchers on the twin dataset, and found that, under ideal imaging conditions, twins could generally be identified well, with one matcher obtaining a EER of only 0.01 . However, when testing these matchers under non-ideal imaging conditions including expression variation, lighting variation, and facial occlusion from glasses, all the matchers saw significant performance decreases. A similar study from Paone et al. [1] further examined the performance of several face recognition algorithms on the Twins Days dataset. This work examined the effects of illumination variation, expression variation, age, and gender on twin face recognition, for images taken on the same day as well as cross year matches in which data from one year was used as the gallery, and the other year the probe. This study produced similar results to others [8], [9], in that, under ideal imaging conditions, twins can be recognized, but imaging non-idealities significantly reduce performance. The worst results in this study were found in the cross-year experiments in which every algorithm performed worse than when presented with images taken on the same day. These experiments showed notable difficulty in differentiating between identical twins using images taken in two separate years than those taken on the same day. This is a particularly important result because, in many real-world FR applications, a face image could potentially be used as a gallery image for the recognition of an identity for several years.

Multiple works have also explored using low-level facial features to identify identical twins. In an early work from Klare et al. [10], authors identify three levels of facial features that, when fused, can improve the facial recognition performance of identical twins. The work extracted multi-scale local binary patterns, scale-invariant feature transform features, and manually 
annotated facial marks from identical twin face images which were broken into face components (eyes, eyebrows, mouth, and nose). The results of the work indicate that the extraction and fusion of these features and face components led to better recognition performance of identical twins. A later study of component-based facial recognition of identical twins from Mahalingam and Ricanek [11] confirmed the results of previous work by again showing that the fusion of facial components of identical twins improves facial recognition performance. Additionally, this study analyzed the impact of age and gender-based differences on facial recognition. Using the framework developed in the study, the authors found that gender made no significant impact to recognition performance, while age showed a positive impact on performance as the twin pairs age.

\subsubsection{Facial Recognition of Look-alikes}

Another of the most challenging cases presented to facial recognition systems is that of look-alike faces. An early study from Kosmerlj et al. [12] on the robustness of a facial recognition tool to the occurrence of look-alikes in the general public found that the tested tool would not be robust to look-alikes. This study also reported an estimate of the frequency of look-alikes in the general public via the number of false accepts returned for each subject in the two tested datasets (labeled as dataset II and dataset III in the work). In the experiments performed the authors found that $97 \%$ and $99.99 \%$ of the subjects in datasets II and III respectively had one or more false accepts at a false accept rate of $1 \%$. The experiments were repeated at a false accept rate of $0.1 \%$ and found that dataset II showed almost no subjects with one or more false accepts, but dataset III showed $92 \%$ of subjects having one or more false accepts. The authors admit that this evaluation may overestimate the true occurrence of look-alikes due to the overall performance of the facial 
recognition tool used in the study. In a preliminary study of look-alike facial recognition [13], researchers developed a dataset of carefully constructed look-alike pairs. The results of this work showed that several facial recognition tools that were state-of-the-art at the time of publication performed poorly on this look-alike dataset. In a second study of look-alike recognition using the dataset constructed in [13], Sun et al. [2] showed that even neural-network-based FR approaches struggle with look-alike recognition. This work developed a deep Siamese convolutional neural network for twin recognition and found that both twins and look-alikes continue to pose significant challenges to the most advanced facial recognition techniques. A very recent work from Rathgeb et al. [14] confirmed the conclusions of previous work in this sphere by showing the negative effect of look-alikes on even the most advanced facial recognition tools. This work developed a handpicked dataset of tailored look-alike pairs and tested the effectiveness of several facial recognition tools on this dataset. The results of this study show that four of the current state of the art facial recognition tools have very high imposter attack presentation match rate (IAPMR) when presented with a dataset containing look-alikes. This led the authors to the conclusion that these tools would not be robust to look-alike occurrences in general.

\subsubsection{Facial Similarity}

A final related topic is that of facial similarity. Previous works have identified facial similarity as a topic that is distinctly different from facial recognition. Wherein facial recognition is used to differentiate between distinct identities, facial similarity is designed to calculate the similarity of the faces of two identities. One of the earliest works on facial similarity [15] laid the foundation of similarity as distinct from facial recognition, and developed a methodology to determine facial similarity using an eigenfaces framework. This study investigated facial similarity 
in a dataset of face images with illumination variation, occlusion, and pose variation, and ultimately used the similarity score returned by the eigenfaces tool to rank the most similar face images to a probe image via the generated similarity score. A more recent work from Sadovnik et al. [16] further developed the concept of facial similarity, and trained a deep neural network to rank similar faces in large scale datasets. This work used a dataset of human-chosen similar face images to train a deep neural network to return high similarity scores for visually similar faces via triplet loss. This work also studied the difference between facial similarity and facial recognition by testing the developed similarity network as a facial recognition tool. The authors found that their look-alike network performed worse at facial recognition than the VGG network the lookalike network was based on. The authors used this fact as evidence that their look-alike network had indeed been trained to complete a distinct task, that of determining facial similarity. Additionally, the determination of facial similarity has been shown to have practical applications in other use cases. A recent work from Rottcher et al. [17] used the concept of facial similarity to find appropriate look-alike pairs for the generation of morphed faces. This work employed a variety of distinct features to determine the similarity of the faces used in the study and proved that their intelligently selected morphed faces out-performed randomly selected morphed pairs.

\subsection{Goals and Objectives}

The primary goal of the research effort presented here is to quantify the facial similarity of identical twins in general. This similarity measurement represents the worst-case baseline of facial similarity in any non-mated comparison in facial recognition. In addition to this baseline measurement, a method of quantitatively determining facial similarity must be developed in order to determine the worst-case baseline measurement. Finally, match experimentation will be carried 
out to demonstrate the effect of highly similar faces on both commercial off the shelf and academic machine learning based facial recognition tools and identify potential look-alike identities.

The tasks required to successfully complete the goals laid out in this work are as follows. First, face matching experimentation must be carried out to demonstrate the effect of identical twin faces on two facial recognition tools. This task will evaluate both identical twin datasets and largescale non-twin datasets to build upon previous work's evaluation of the effect of identical twin and look-alike faces and identify potential look-alike pairs in the large-scale non-twin datasets. Second, a method of quantitatively determining the facial similarity of any two faces must be developed. This measure should be based on the facial similarity of identical twins, as twins represent the worst-case scenario of facial similarity in facial recognition. This measure must directly compare two faces and return a quantitative measure of how similar the faces are (i.e., a similarity score), not a comparison score between the faces. This measure will be based on a deep convolutional neural network designed to directly compare two face images. Third, the similarity measure must be evaluated using both twin and non-twin datasets. This evaluation will determine the worst-case baseline measure of facial similarity, identify highly similar faces within the non-twin datasets, and serve as a point of comparison between the similarity score and comparison score returned by facial recognition tools. Additionally, this evaluation will provide an estimate of look-alike identities in a large-scale non-twin face dataset.

\subsection{Impact}

If successful, this work will have impacts on facial recognition at large via its contribution of a worst-case baseline of facial similarity in human facial recognition. Additionally, this work will demonstrate a deep convolutional neural network designed to determine the facial similarity 
of any two faces, which has several useful applications. The most important application of this method is finding non-mated look-alikes in large scale datasets. This can be used to estimate the number of look-alike identities in a dataset or in the human population given a large enough and suitably representative dataset. The look-alike identities found via this method could also be used to generate high quality morphed faces. The proposed similarity network also provides a method of relating the comparison score returned by a facial recognition tool to the similarity score returned by the network. This is a useful observation, as it allows for the investigation of the relationship between a comparison score, which may not be directly correlated to facial similarity, and a similarity score based directly on the facial similarity of two faces in comparison. Additionally, this work builds upon previous work in this sphere to further highlight the difficulty posed to automatic facial recognition systems by both identical twin and look-alike pairs. 


\section{Chapter 2: Theory and Background}

\subsection{Facial Recognition Theory}

Automatic facial recognition systems have achieved remarkable accuracy in recent history, with several state-of-the-art algorithms achieving better than human performance on difficult face recognition tasks. ArcFace [18], a current state-of-the-art face recognition neural network achieved 99.83\% verification accuracy on the popular LFW face dataset [19]. Despite these advancements in face recognition technology and performance, these approaches still face difficulty with the hardest cases in facial recognition.

In most facial recognition applications, there are two primary types of comparisons performed between face images. These are mated or genuine comparisons and non-mated or impostor comparisons. In a mated comparison two face images containing the same individual are compared, while in non-mated comparisons two face images containing different identities are compared. When performing facial recognition experiments, it is expected that the comparison score from a mated comparison of a given identity will be higher than that of a non-mated comparison for the same identity.

Given the idea that a mated comparison will produce a higher comparison score than a nonmated comparison, it is then possible to introduce the concept of a biometric comparison decision. ISO/IEC Standard 2382-37 [20] defines a comparison decision as "determination of whether the biometric probe(s) and biometric reference(s) have the same biometric source, based on a comparison score(s), a decision policy(ies) including a threshold, and possibly other inputs". This definition shows that the purpose of a comparison decision is to determine whether a biometric comparison is mated or non-mated, this is also known as determining whether a comparison is a 
match or non-match. A match is a result of a comparison decision indicating that the two compared samples belong to the same identity, and a non-match is a result indicating that the samples belong to different identities. The comparison decision can be determined by a variety of factors but is often made using a threshold designed to separate mated and non-mated comparisons via their comparison scores. A threshold works in such a manner to separate a distribution of comparison scores into the two categories match and non-match, where scores higher than the threshold are considered matches and scores lower than the threshold are considered non-matches. While the goal of determining a comparison decision is to accurately classify samples into match and nonmatch categories, it is a fact that classification errors happen when making comparison decisions. This leads to the definition of erroneous results of comparison decisions, the false match and the false non-match. A false match occurs when two samples belonging to different identities are determined to be a match in a comparison decision. A false non-match is the inverse of this in which two samples belonging to the same identity are determined to be a non-match in a comparison decision. Generally, the threshold for comparison decisions is tuned to produce optimal classification accuracy, however this can be very situation dependent. In some cases, it is vital that no false matches occur, but the number of false non-matches is irrelevant. Similarly, there may be a use case in which the number of false matches that occur is unimportant, but the existence of false non-matches is unacceptable. In both example cases the threshold that is tuned for optimal comparison decision accuracy would not be an acceptable threshold for the application. A general rule for deciding a comparison decision threshold is to examine both the mated and non-mated comparison score distributions for a representative population for the use case. By examining the two distributions a threshold can be determined by choosing the approximate location on the axis 
of comparison scores where the two distributions intersect, an example of this is shown in Figure 3.

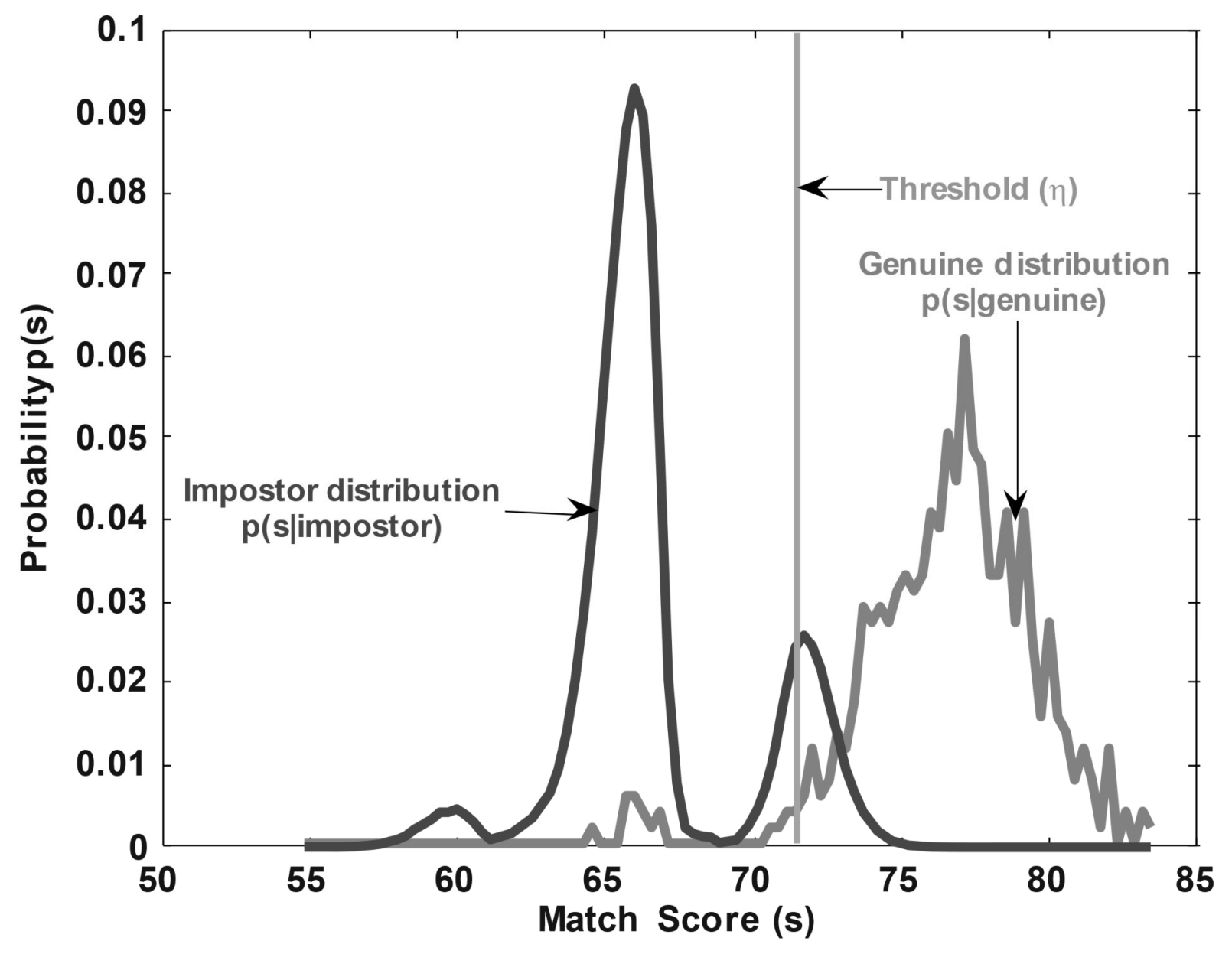

Figure 3: Example of comparison decision threshold [21].

The performance of facial recognition systems can be judged in several ways. The most basic of these metrics is the simple probability that any two samples will be misclassified. Separated into two cases, the false match rate and false non-match rate (AKA false accept rate and false reject rate), these two metrics present the probability of any two non-mated samples being misclassified as a match and any two mated samples being misclassified as a non-match, 
respectively. As these metrics demonstrate the probability of misclassification lower values indicate better performance. Both metrics can be combined to demonstrate the performance of a facial recognition system at a range of thresholds through the receiver operating characteristic (ROC) of the system. In the determination of the receiver operating characteristic the threshold used to determine the outcome of a comparison decision between two samples is varied over a range of values (typically the entire range of match scores) at even intervals for the comparison score distributions in question. At each threshold step the false match rate and false non-match rate is calculated and this value is then plotted on a two-dimensional graph consisting of false match rate and false non match rate axes. This step is repeated for all threshold values, with the result consisting of a two-dimensional curve which indicates the recognition performance of the facial recognition tool at a range of possible threshold values. Additionally, the area under the receiver operating curve (often referred to as AUC) is a further performance metric which captures the overall performance of the recognition tool at any threshold value. A higher value for the area under the curve indicates better overall classification performance. A final performance metric commonly used for facial recognition systems is the equal error rate (EER). In Introduction to Biometrics [21], Jain et al. state "The EER refers to that point in a DET (or ROC) curve where the FAR equals the FRR; a lower EER value, therefore, indicates better performance.". The performance metric used to show the effectiveness of a facial recognition system varies by use case, but area under the curve and equal error rate are two popular metrics for demonstrating the overall performance of a facial recognition tool.

Facial recognition experiments focus on the comparison of face photos from one or many individuals. In general, there are two types of experiments, those being: one-to-one experiments (AKA verification), and one-to-many experiments (AKA identification). In one-to-one 
experiments, the goal of the experiment is to verify the identity of an individual by comparing a sample from that individual to a different sample from either a different identity or the same identity. These comparisons often occur in access control applications and are used primarily to confirm the identity of an individual. The second type of comparison, one-to-many, involves comparing a sample from one individual to a collection of samples from many different identities. The single sample that is compared against the rest of the samples is referred to as the probe, and the collection of samples which that sample is compared against is known as the gallery. The identities contained within the gallery may or may not contain a different sample from the probe identity, but in all cases, the same probe sample must not be contained within the gallery. One-tomany comparison is often used in biometric search applications, wherein the probe comes from an unknown identity, and it is compared against a gallery of known identities to attempt to find a match.

\subsection{Facial Recognition Tools}

Over the long history of automated facial recognition, there have been a litany of techniques introduced to solve the problem of accurately identifying humans via facial biometrics. The field has grown substantially from the first fully automatic facial recognition technique introduced by Kanade in [22], which employed edge detection and a technique known as integral projection to automatically extract a predefined set of facial landmark points from face images which could then be used for identification. From this point forward many other facial recognition approaches were introduced. These methods include: face feature or landmark based approaches, principal component analysis (PCA) based approaches (Eigenfaces) [23], linear discriminant analysis (LDA) based approaches (Fischerfaces) [24], and neural network-based approaches [18], 
[25], [26]. A common thread between each of these recognition techniques is that all of them have difficulty handling both identical twin and look-alike images.

Feature or landmark based facial recognition approaches are the origin of automated facial recognition. Originally introduced as man-machine methods, the first facial recognition approaches used human marked facial feature locations which were then fed to a computing system to identify subjects. As mentioned above, this idea was converted to a fully automated process in [22]. Feature based recognition techniques are still employed today and are commonly the basis of commercial off the shelf facial recognition tools. All these techniques share the framework of first extracting a set of predetermined facial features found on all faces from a given face. The set of facial features that is extracted varies between methods but is generally chosen to maximize the recognition performance of the algorithm. Next, the set of extracted features is compared mathematically to the extracted features of another face to determine the comparison score for the face pair in question. The comparison method used also varies between techniques but is commonly a form of distance comparison between the feature points on the faces. These methods struggle with twin and look-alike images because the features extracted from these faces are generally in the same position and are the same or similar shape and size, especially within identical twin pairs.

Principal component analysis and linear discriminant analysis facial recognition techniques (Eigenfaces [23] and Fischerfaces [24] respectively) both employ the same general idea of projecting a given face image onto a subspace in which facial recognition can be performed. Described as appearance-based techniques in Introduction to Biometrics, Jain et al. say "Appearance-based schemes are based on the idea of representing the given face image as a function of different face images available in the training set, or as a function of a few basis faces." 
[21]. Both these methods employ eigenvalue decomposition to create an eigenspace from a dataset of faces in which new face image vectors can be projected onto to allow for Euclidean distancebased classification between the projected faces. The chief difference between the two methods is that the Fischerfaces method employs the class label of the image (i.e., the identity information of the individual contained within the face image) in its creation of the subspace to maximize the separability of the classes when projected onto the subspace. These methods fall short when presented with identical twin and look-alike face images due to the similarity of the faces, and the resultingly similar subspace projections.

The newest development in facial recognition technology has been the application of neural networks to the task. These facial recognition tools have shown impressive accuracy on the largest face datasets ever created and have even been able to address challenging problems such as pose and lighting variance. Neural network based facial recognition approaches vary in the architecture and structure used, but they all rely on the use of the convolutional neural network as a foundation for their operation. The convolutional neural network acts in a similar manner to a traditional feed forward neural network but applies convolution filters to the input as it is passed to each subsequent layer. The output of the convolution filters at each layer are the input to the following hidden layer, and through the training of the network the filter kernels are learned just as the weights are learned in a traditional neural network. The primary way in which neural networks have been used for face recognition in recent years is to directly learn a subspace representation of the input face image. This approach attempts to distill the input face image into a representative feature vector known as an embedding. Approaches using this methodology have gained popularity over the years due to their innate ability to handle new identities presented to the network for classification in the socalled "open set" face classification problem. This approach employs the convolution neural 
network to reduce the input face image into a face embedding which represents the features of the input face. This embedding can then be used for classification via several methods including distance-based comparisons or other linear classifiers such as support vector machines. Ideal facial recognition tools that take this approach aim to minimize the intra-class distance and maximize the inter-class distance within the embedding space to maximize recognition performance. While these networks are the most powerful facial recognition tools created to date, they still face challenges when presented with look-alike and identical twin images. This is again due to the similarity of the faces in question, causing classification errors because of the closeness of the face embeddings of these face pairs in the embedding space.

\subsection{Neural Networks Theory}

Machine learning, particularly neural networks have become a widely discussed and researched topic in the past decade. Initially stemming from the idea to construct an algorithm that functions in the same fashion as the human mind, neural networks seek to solve challenging problems by processing data through a series of interconnected nodes within the algorithm known as neurons. This structure allows for the algorithm to learn by observing the data passed to it, with the end goal of improving the performance on the required task.

As far back as the 1940s scientists were already conceiving the idea of a mathematical representation of biological neurons as binary logic nodes [27]. This idea was extended further by Hebb, in which he conceived of the idea of a network of neurons becoming stronger and learning a task through repeated exposure to this task [28]. This idea became known as Hebbian learning, and was soon transferred to the world of computing with the development and introduction of the perceptron in the late 1950s by Rosenblatt [29]. The field of artificial neural networks ebbed after 
the introduction and further development of the perceptron, as researchers believed that the usefulness of these tools was limited, but with the later re-introduction and application of concepts such as backpropagation and gradient descent to neural networks in the 1980s interest in the concept was renewed. These two ideas, along with the massive increase in computing power seen over the gap in interest surrounding neural networks, allowed for the development of deeper and more complex networks. These new networks were able to tackle difficult tasks simply by learning from the data passed to them. Ever since, neural networks have been applied to nearly every conceivable machine learning task, with research surrounding neural networks and their applications being at an all-time high.

Neural networks are well suited to many tasks, one of which is of chief importance to this work is image classification. The typical network used for this task is the convolutional neural network. As discussed previously this network type contains convolutional filters at each of its convolutional neurons within the network architecture. These networks function in much the same manner as a traditional multi-layer feed forward network, with the network learning to optimize the filter kernels at each of its neurons. LeCun et al. in [30] state "At the output, each feature map represents a particular feature extracted at all locations on the input.", here a feature map refers to the output of a convolution filter. As described, this optimization allows for the network to learn directly from the content of input images at different scales which are defined by the size of convolution filters used in the architecture. An example convolutional neural network architecture is shown in Figure 4. 


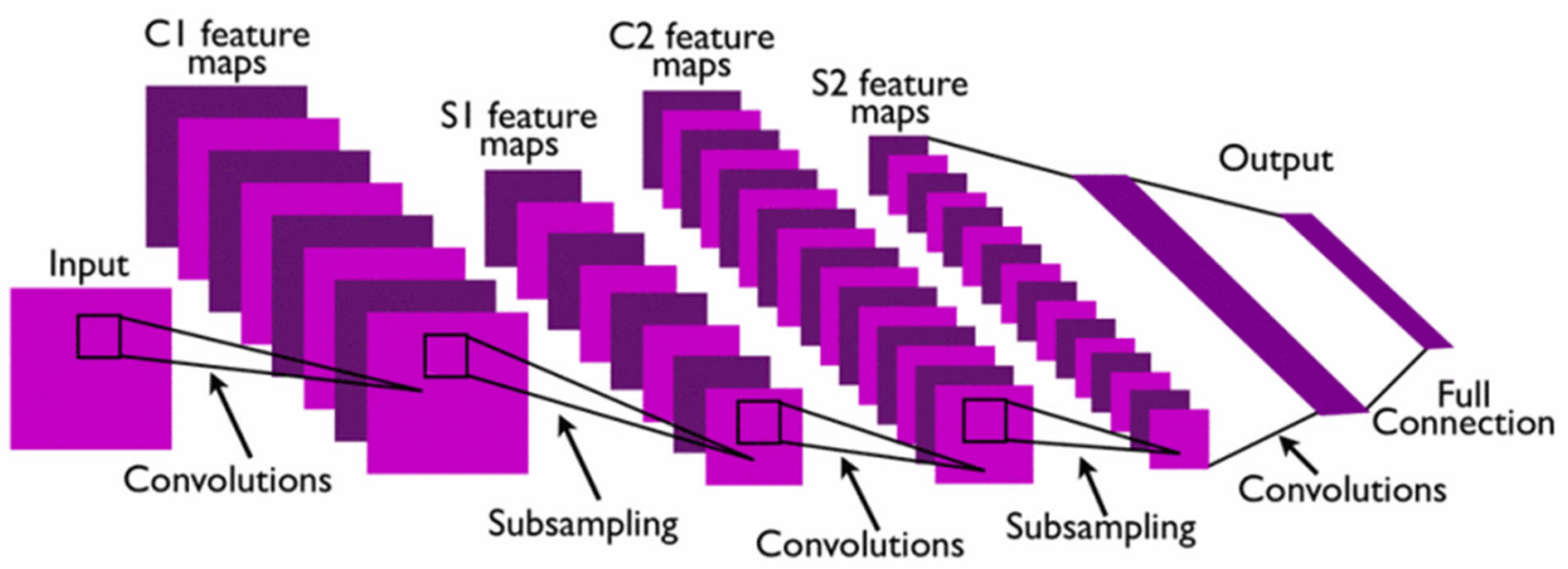

Figure 4: An example convolutional neural network architecture [30].

This network type also frequently employs a layer known as the max-pooling layer. This layer serves as a dimensionality reduction layer which down samples the input image based on the maximum value found within a defined region of the image, allowing for further learning from features at various scales within the image. A visual representation of max pooling is found in Figure 5.

\begin{tabular}{|c|c|c|c|}
\hline 12 & 20 & 30 & 0 \\
\hline 8 & 12 & 2 & 0 \\
\hline 34 & 70 & 37 & 4 \\
\hline 112 & 100 & 25 & 12 \\
\hline
\end{tabular}$\stackrel{2 \times 2 \text { Max-Pool }}{\longrightarrow}$\begin{tabular}{|c|c|c|}
\hline 20 & 30 \\
\hline 112 & 37 \\
\hline
\end{tabular}

Figure 5: Visual representation of max-pooling step [31]. 
Convolutional neural network architectures are generally terminated by fully connected bottleneck layers. These layers serve to translate the processing done by the preceding layers into a useful output, such as a predicted class vector or an extracted feature embedding vector. One of the most well-known convolutional neural networks AlexNet [32], used these bottleneck layers to predict an input image as belonging to one of 1000 possible classes. The FaceNet architecture discussed in the next section employs its bottleneck layers to return a representative feature embedding of any given input face image.

As neural networks learn directly from input training data, it is advantageous to use these methods for tasks involving large quantities of data. Especially as datasets continue to grow, these tools will serve as important methods of solving difficult machine learning tasks into the future.

\subsection{FaceNet}

The proposed similarity network developed in this research is built upon the FaceNet neural network introduced by Schroff et al. in the paper "FaceNet: A Unified Embedding for Face Recognition and Clustering" [26]. At the time of publication this facial recognition tool produced state-of-the-art results on the most difficult face datasets at the time, particularly due to the network's pose and lighting invariant face embeddings. The network developed in this work directly learns a representation of the input face known as the face embedding. The output of the network is structured such that the generated face embeddings exist in an embedding subspace such that intra-class embeddings reside close to one another, and inter-class embeddings reside far apart, which leads to accurate classification through even simple distance-based measurements between face embeddings. 
The FaceNet architecture relies heavily on the inception layer architecture first introduced by Szegedy et al. in [33]. The inception layer is a unique convolutional neural network layer, as instead of containing only one bank of convolution filters, it passes the input to convolution filters of multiple sizes. The most basic of these layers uses three different convolution filter sizes (1x1, $3 \times 3$, and $5 \times 5)$ and concatenates the output of these three filter sizes as the input to the following layer. Other versions of the inception layer implement max-pooling within the block to further reduce the dimensionality of the input image. The primary benefit of these layers is the increase in computational performance provided by their use. By being able to process features of different sizes within a single convolutional layer of the network, the network architecture can become wider versus deeper, leading to better computational performance and avoiding many of the pitfalls commonly seen in very deep convolutional neural network architectures. The best performing network architecture developed in the FaceNet publication (called NN2 in the work) was almost identical to the GoogLeNet architecture developed in [33], which uses almost exclusively inception layers in its architecture, Table 1 demonstrates this architecture. 
Table 1: FaceNet NN2, neural network architecture.

\begin{tabular}{|c|c|c|c|c|c|c|c|c|c|c|}
\hline type & $\begin{array}{l}\text { output } \\
\text { size }\end{array}$ & depth & $\# 1 \times 1$ & $\begin{array}{l}\# 3 \times 3 \\
\text { reduce }\end{array}$ & $\# 3 \times 3$ & $\begin{array}{l}\# 5 \times 5 \\
\text { reduce }\end{array}$ & $\# 5 \times 5$ & $\begin{array}{l}\text { pool } \\
\text { proj (p) }\end{array}$ & params & FLOPS \\
\hline conv1 $(7 \times 7 \times 3,2)$ & $112 \times 112 \times 64$ & 1 & & & & & & & $9 \mathrm{~K}$ & $119 \mathrm{M}$ \\
\hline $\max$ pool + norm & $56 \times 56 \times 64$ & 0 & & & & & & $\mathrm{~m} 3 \times 3,2$ & & \\
\hline inception (2) & $56 \times 56 \times 192$ & 2 & & 64 & 192 & & & & $115 \mathrm{~K}$ & $360 \mathrm{M}$ \\
\hline norm + max pool & $28 \times 28 \times 192$ & 0 & & & & & & $\mathrm{~m} 3 \times 3,2$ & & \\
\hline inception (3a) & $28 \times 28 \times 256$ & 2 & 64 & 96 & 128 & 16 & 32 & $\mathrm{~m}, 32 \mathrm{p}$ & $164 \mathrm{~K}$ & $128 \mathrm{M}$ \\
\hline inception (3b) & $28 \times 28 \times 320$ & 2 & 64 & 96 & 128 & 32 & 64 & $L_{2}, 64 \mathrm{p}$ & $228 \mathrm{~K}$ & $179 \mathrm{M}$ \\
\hline inception (3c) & $14 \times 14 \times 640$ & 2 & 0 & 128 & 256,2 & 32 & 64,2 & $\mathrm{~m} 3 \times 3,2$ & $398 \mathrm{~K}$ & $108 \mathrm{M}$ \\
\hline inception (4a) & $14 \times 14 \times 640$ & 2 & 256 & 96 & 192 & 32 & 64 & $L_{2}, 128 \mathrm{p}$ & $545 \mathrm{~K}$ & $107 \mathrm{M}$ \\
\hline inception (4b) & $14 \times 14 \times 640$ & 2 & 224 & 112 & 224 & 32 & 64 & $L_{2}, 128 \mathrm{p}$ & $595 \mathrm{~K}$ & $117 \mathrm{M}$ \\
\hline inception (4c) & $14 \times 14 \times 640$ & 2 & 192 & 128 & 256 & 32 & 64 & $L_{2}, 128 \mathrm{p}$ & $654 \mathrm{~K}$ & $128 \mathrm{M}$ \\
\hline inception (4d) & $14 \times 14 \times 640$ & 2 & 160 & 144 & 288 & 32 & 64 & $L_{2}, 128 \mathrm{p}$ & $722 \mathrm{~K}$ & $142 \mathrm{M}$ \\
\hline inception $(4 \mathrm{e})$ & $7 \times 7 \times 1024$ & 2 & 0 & 160 & 256,2 & 64 & 128,2 & $\mathrm{~m} 3 \times 3,2$ & $717 \mathrm{~K}$ & $56 \mathrm{M}$ \\
\hline inception (5a) & $7 \times 7 \times 1024$ & 2 & 384 & 192 & 384 & 48 & 128 & $L_{2}, 128 \mathrm{p}$ & $1.6 \mathrm{M}$ & $78 \mathrm{M}$ \\
\hline inception (5b) & $7 \times 7 \times 1024$ & 2 & 384 & 192 & 384 & 48 & 128 & $\mathrm{~m}, 128 \mathrm{p}$ & $1.6 \mathrm{M}$ & $78 \mathrm{M}$ \\
\hline avg pool & $1 \times 1 \times 1024$ & 0 & & & & & & & & \\
\hline fully conn & $1 \times 1 \times 128$ & 1 & & & & & & & $131 \mathrm{~K}$ & $0.1 \mathrm{M}$ \\
\hline L2 normalization & $1 \times 1 \times 128$ & 0 & & & & & & & & \\
\hline total & & & & & & & & & $7.5 \mathrm{M}$ & $1.6 \mathrm{~B}$ \\
\hline
\end{tabular}

The FaceNet network was trained using the triplet loss function which aims to encourage the network to reduce the distance between samples of the same class and push apart samples of different classes in the embedding space. A visual representation of this idea is shown in Figure 6.

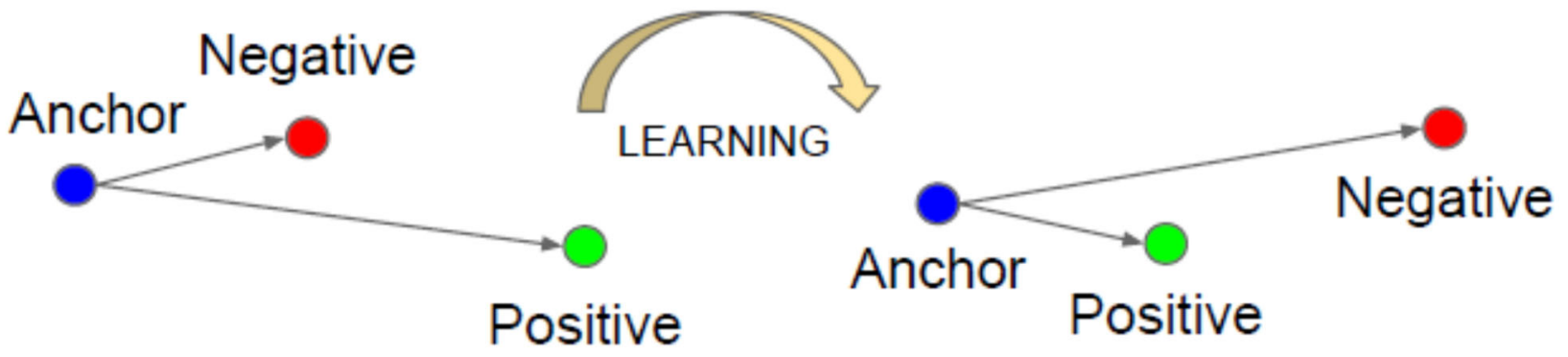

Figure 6: Visual representation of the triplet loss function [26]. 
As shown above, Figure 6 includes three different types of data samples, the anchor, the positive, and the negative. The anchor is the target sample, the positive is a sample of the class as the anchor, and the negative is a sample of a different class than the anchor. These three sample types work in harmony to construct a feature space in which class separability is maximized. Mathematically triplet loss $L$ is defined in [26] as:

$$
L=\sum_{i}^{N}\left[\left\|f\left(x_{i}^{a}\right)-f\left(x_{i}^{p}\right)\right\|_{2}^{2}-\left\|f\left(x_{i}^{a}\right)-f\left(x_{i}^{p}\right)\right\|_{2}^{2}+\alpha\right]
$$

Equation 1: Triplet loss equation.

where $f\left(x_{i}^{a}\right)$ is the anchor embedding, $f\left(x_{i}^{p}\right)$ is the positive embedding, $f\left(x_{i}^{n}\right)$ is the negative embedding, and $\alpha$ is the margin. The margin, $\alpha$, exists to enforce a minimum distance between the positive and negative sample pairs in the embedding space. Within triplet loss there exist three categories of triplets, the easy negative, the semi-hard negative, and the hard negative. Each of these categories defines a distinct case for where the negative sample lies within the embedding space in relation to the anchor and positive samples. A visual representation of the triplet categories is shown in Figure 7 below. 


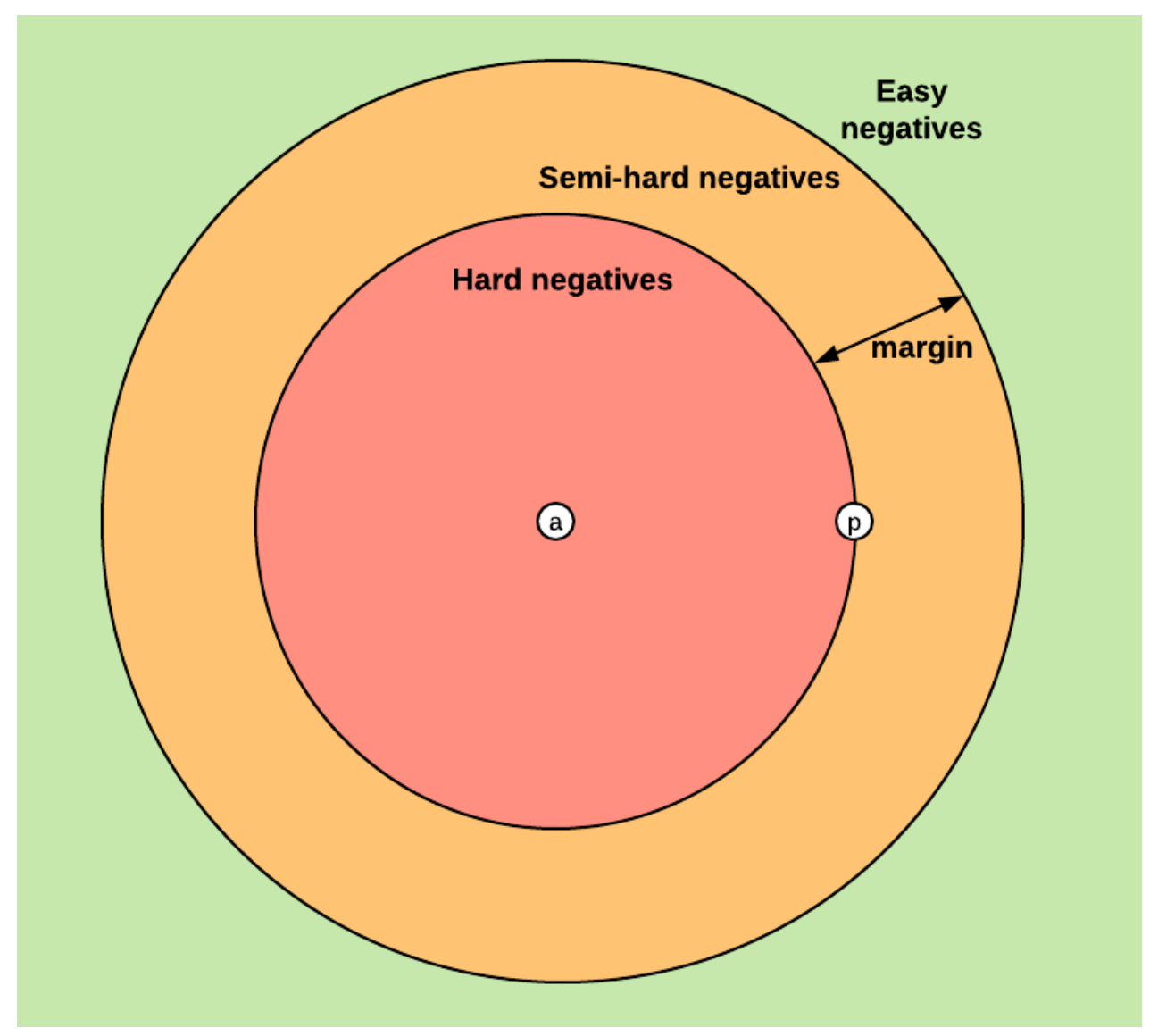

Figure 7: Triplet categories [34].

Easy negatives lie outside the positive sample plus the margin, and do not contribute to the learning of the network, semi-hard negatives lie outside the positive sample but within the margin, and hard negatives lie within the anchor and the positive. Only semi-hard and hard triplets contribute to the learning of the network. As such, the authors of the work discuss the need for the selection of hard and semi-hard triplets, as selecting triplets at random would lead to the occurrence of many easy triplets that do not contribute to the learning of the network. To alleviate this problem, the authors employ an on-line triplet generation process in which hard and semi-hard triplets are chosen from within a mini-batch of the training phase of the network. This led to faster convergence of the network. 
The results and conclusions of this work demonstrate that the method of directly learning a face embedding provides an accurate and efficient way to implement facial recognition using convolutional neural networks. 


\section{Chapter 3: Datasets, Matching}

\section{Experiments, and Similarity Network}

\subsection{Dataset Descriptions}

The face image datasets used in this work come from multiple sources. The most important of these datasets is the twin dataset. This dataset compiles face data from WVU twins days collections from 2010 - 2019. In each of these collections face images were captured at the yearly Twins Days festival using high resolution DSLR imagery. The face images were captured using the SAP 50/51 standard five pose face capture method [35], with even illumination in front of a neutral gray backdrop. This dataset contains 2,269 total identities, with 1,438 of these being identical twin pairs. The rest of the identities in the dataset come from fraternal twin pairs, family members of the twin pairs, and unrelated single individuals. The second dataset used, the non-twin dataset, comes from WVU biometric data collections from 2008-2019 in which ground truth face images were captured. Again, these images were captured using the SAP 50/51 five pose face capture method, with even face illumination and neutral gray backdrop. This dataset contains 5,295 identities, mostly students attending WVU at the time of collection. The final dataset, the large scale non-twin dataset, comes from combining the non-twin dataset with the publicly available CelebA dataset [36]. The CelebA dataset contains images of celebrities scraped from the internet and contains 10,160 identities in total. Images from the CelebA dataset are unconstrained face images taken in-the-wild. 
For the first two datasets, the twin dataset and non-twin dataset, the images were captured using Canon DSLR cameras. Three different cameras were used throughout the collection of the datasets: the Canon 5D Mark II, the Canon 5D Mark III, and the Canon 5DSR. All the images were captured using even illumination, either using $5500 \mathrm{~K}$ tungsten three-point lighting, or $5500 \mathrm{~K}$ LED light panels. Additionally, all the images were captured with the subject positioned in front of a neutral gray backdrop.

The demographics of the twin and non-twin datasets are shown below in Figures 8-13, demographics are not published for the CelebA dataset and are not included here. As shown in the tables, the demographics for both the twin dataset and non-twin dataset are skewed towards Caucasian/white participants. This is a factor of the populations from which biometric data collection participants were selected. 


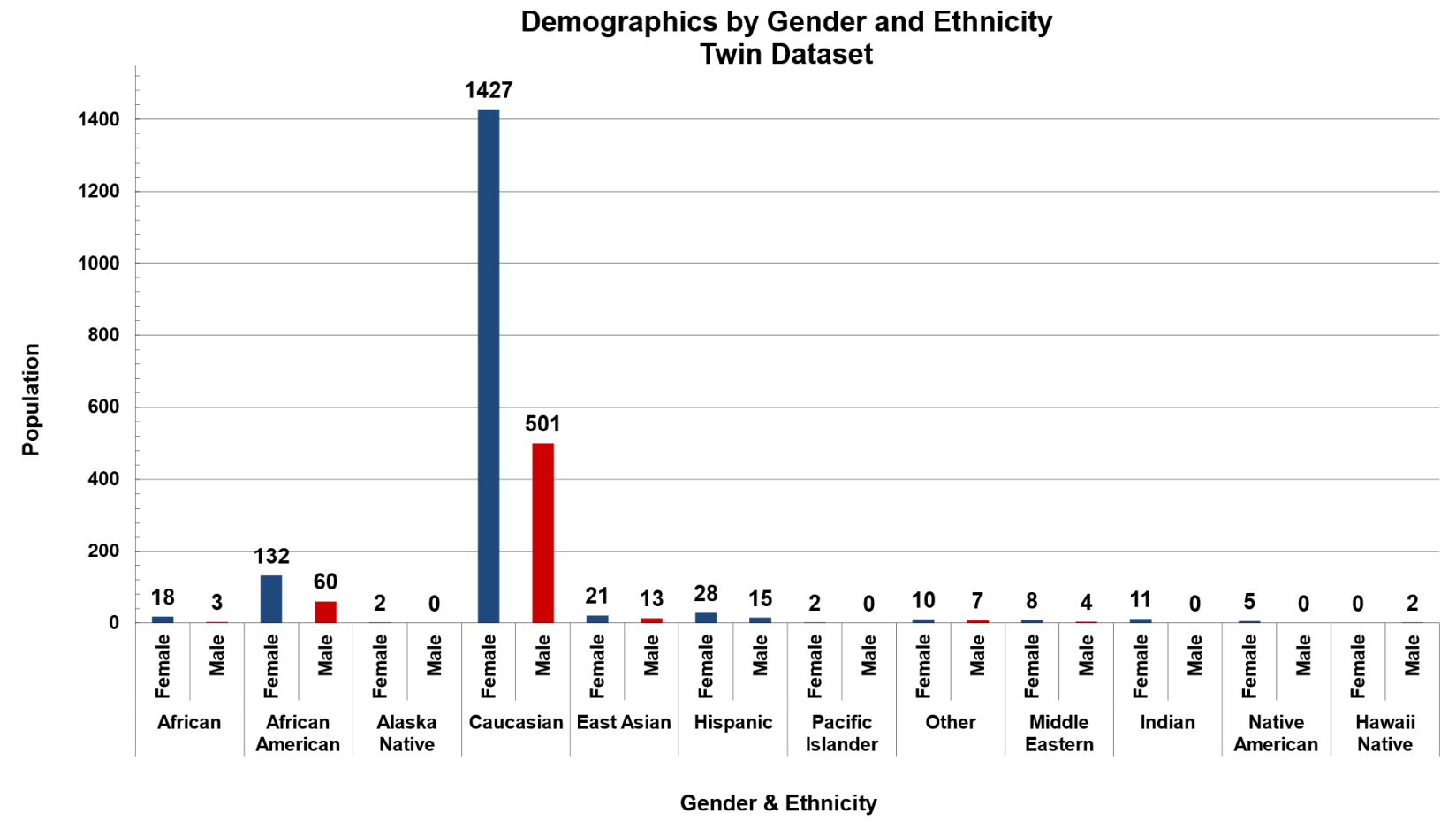

Figure 8: Twin dataset gender and ethnicity demographics chart. 


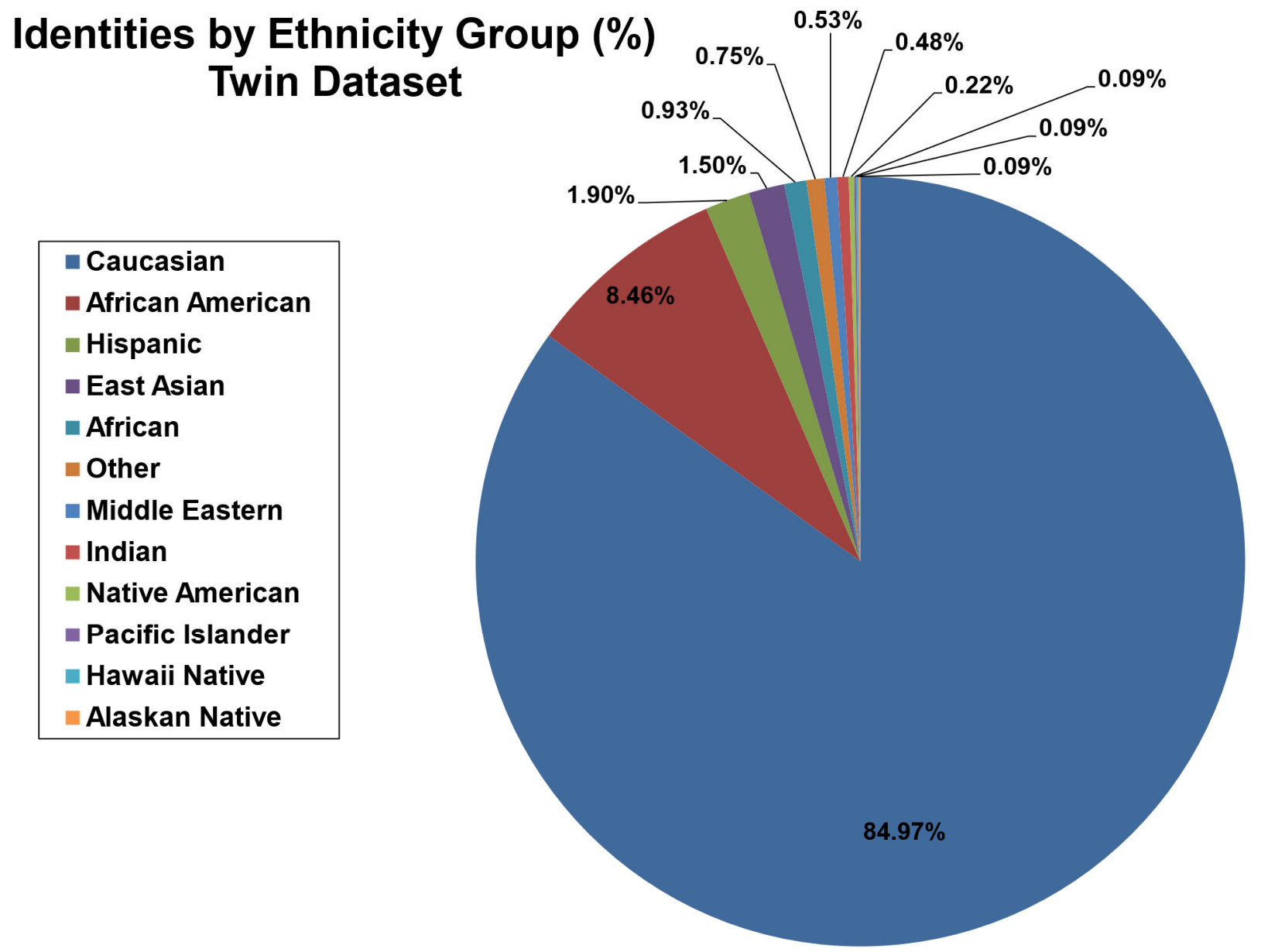

Figure 9: Twin dataset ethnicity demographics chart. 


\section{Identities by Age Group (\%) Twin Dataset}

\begin{tabular}{|l}
\hline$\square 18$ - 19 years old \\
20 - 29 years old \\
$30-39$ years old \\
$\square 0$ - 49 years old \\
$\square 50-59$ years old \\
60 - 69 years old \\
$70-79$ years old \\
$80-89$ years old \\
$90-99$ years old
\end{tabular}

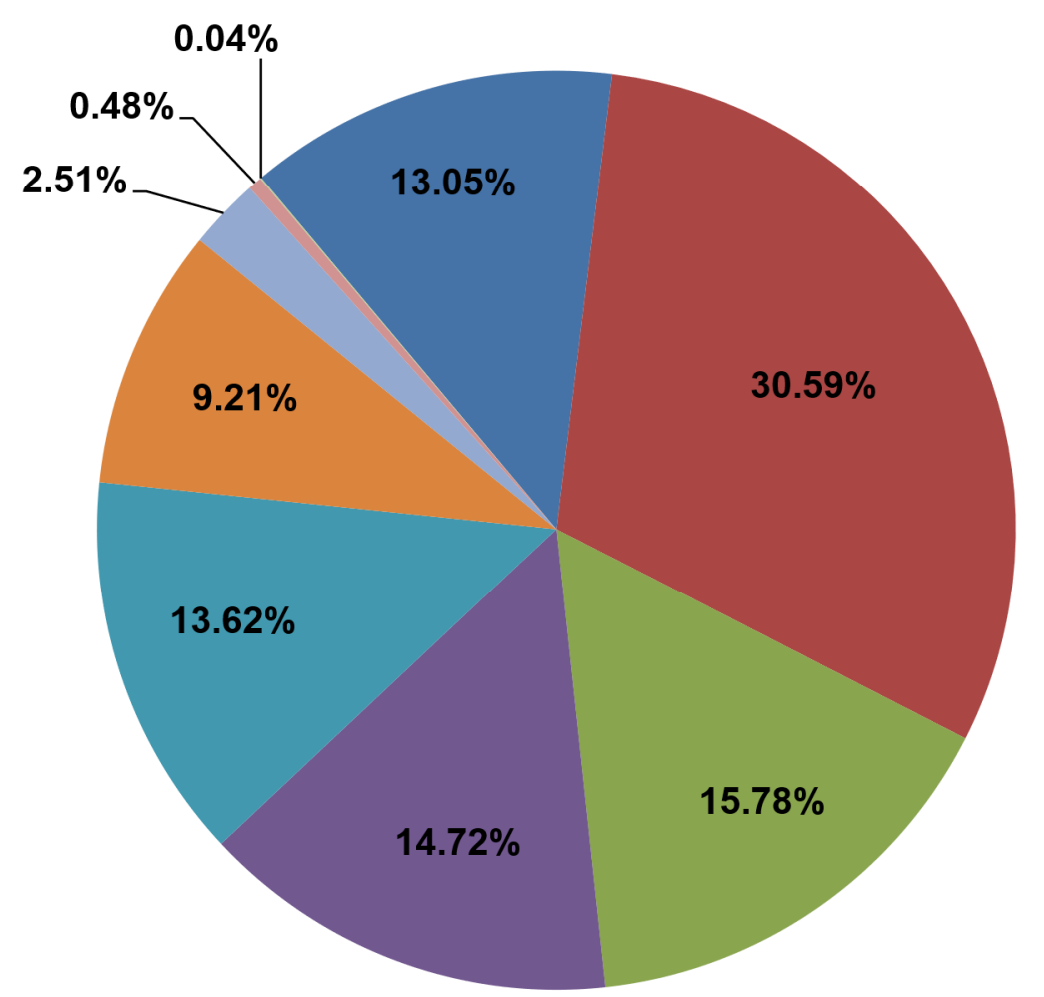

Figure 10: Twin dataset age group demographics chart. 


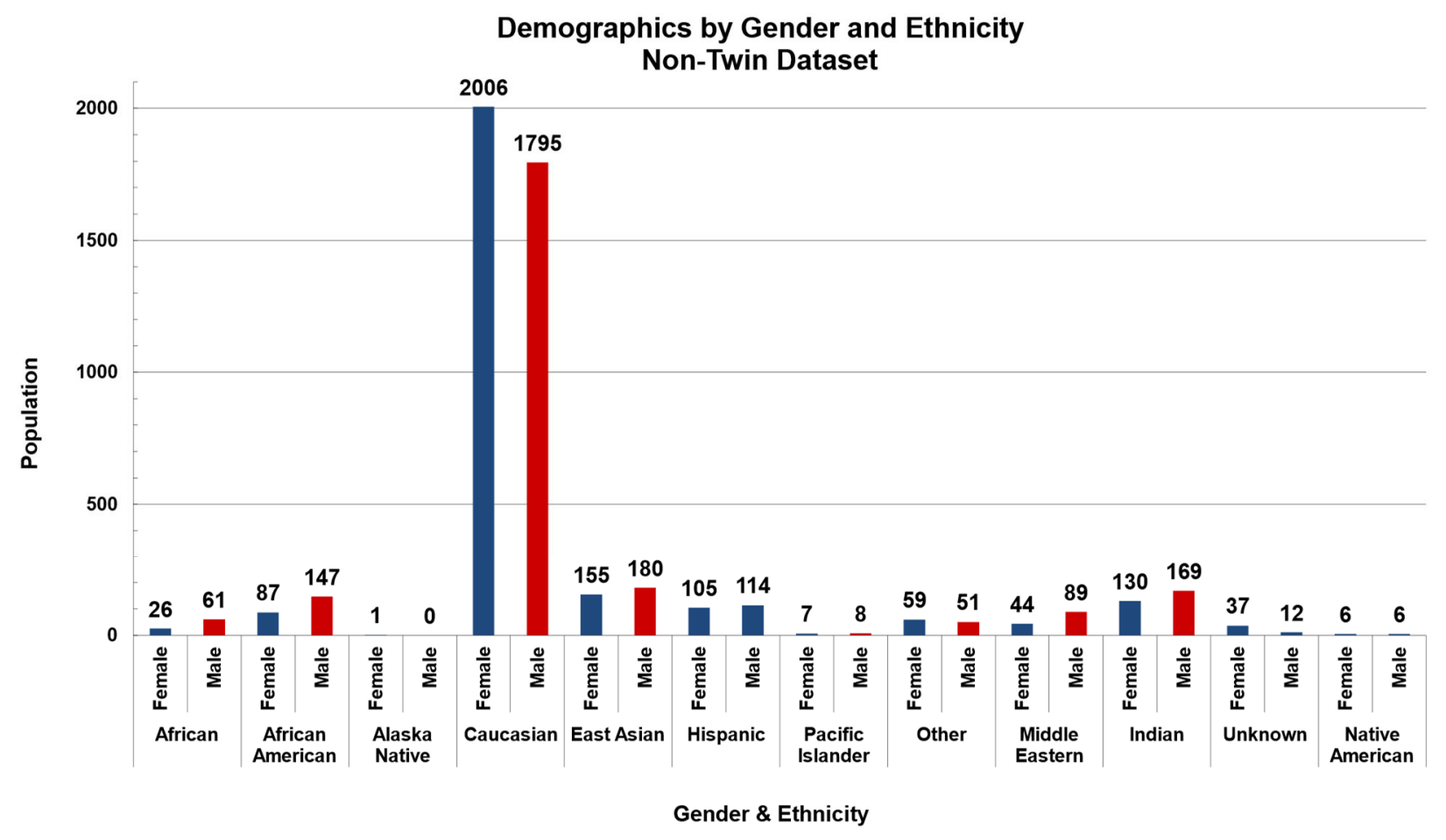

Figure 11: Non-twin dataset gender and ethnicity demographics chart. 


\section{Identities by Ethnicity Group (\%) Non-Twin Dataset}

\begin{tabular}{|l|}
\hline - Caucasian \\
- East Asian \\
- Indian \\
- African American \\
- Hispanic \\
- Middle Eastern \\
- Other \\
- African \\
- Unknown \\
- Pacific Islander \\
- Native American \\
Alaskan Native \\
\hline
\end{tabular}

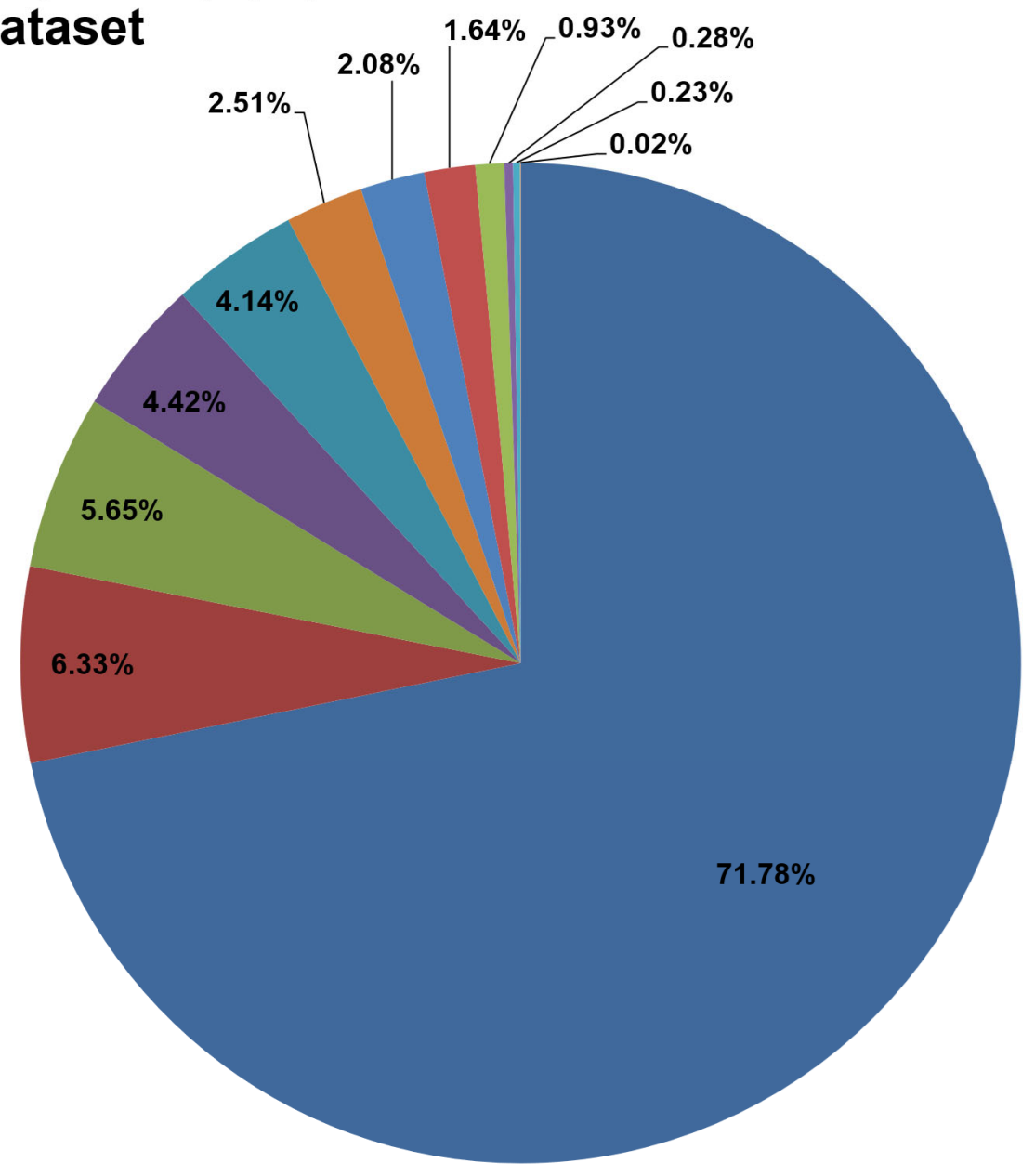

Figure 12: Non-twin dataset ethnicity demographics chart. 


\section{Identities by Age Group (\%) Non-Twin Dataset}
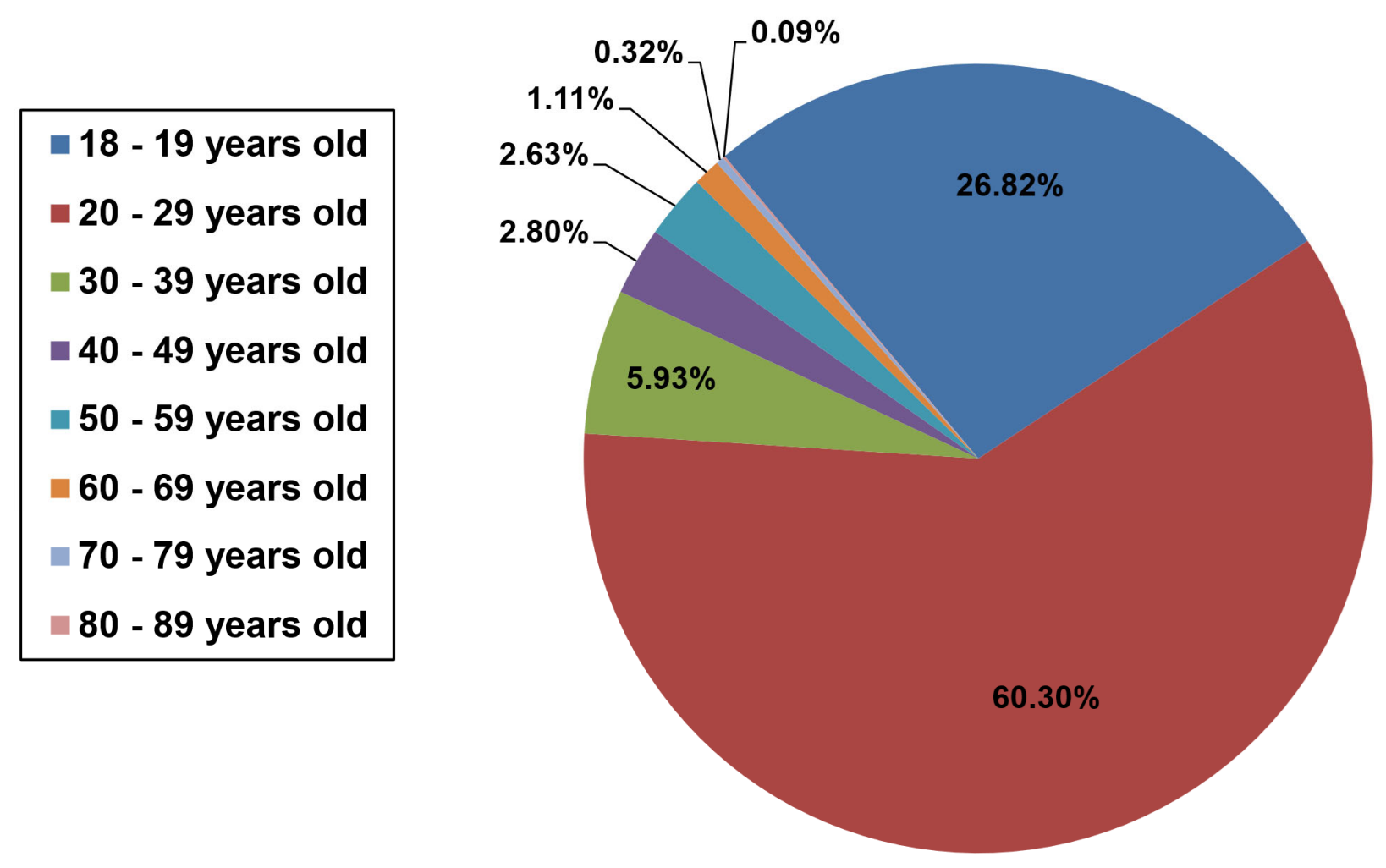

Figure 13: Non-twin dataset age group demographics chart.

Pre-processing for the twin and non-twin datasets involved manual cropping of the face images. These images were cropped to SAP 50 resolution and position standards in three steps. First, the manual selection of facial landmarks (center of eye, tip of nose, and outside edge of the ear) was carried out. Next, image rotation was performed to center and align the facial landmarks to be horizontal in the final cropped image. Finally, the aligned images were cropped around the face to the correct resolution standard. The CelebA dataset was cropped and aligned in a similar method, but performed using a neural network approach known as the Multi-task Cascaded Neural 
Network [37]. This face cropping approach uses neural network architectures to detect the face locations within the images, rotate and align the face, and crop the face from the image based on the detected face position. The CelebA images were cropped to a size of $182 \times 182$ pixels, with a tight margin around the central region of the face.

\subsection{Matching Experimentation}

After compiling and preparing the face datasets, the initial task of the work presented here was to analyze the performance of two facial recognition systems on these datasets. Two FR tools were used in this experiment, the first of which was a commercial off the shelf "black box" matcher, and the second was the FaceNet matcher [26], which is based on the Inception-ResNet v1 architecture. The commercial off the shelf matcher used was the Neurotechnology V4 Verilook matcher [38]. This matcher is a multi-step feature-based matcher which first creates feature templates of a given face image, then compares those feature templates to return a comparison score for the given faces. The exact features used in the template creation and the comparison method used by the matcher are proprietary technology, hence the "black-box" name given to this type of facial recognition tool.

The first experiment performed was a baseline analysis of the matchers when presented with only the identical twin pairs from the twin dataset to determine the effect of highly similar faces on the non-mated distribution of a FR experiment. In addition, a mated comparison was made for each identity to show the relationship between the identical twin non-mated distribution and mated distribution. The mean comparison score of the identical twins in this baseline experiment is used as the threshold for each of the remaining comparison experiments. This score represents an experimental threshold for individuals with high facial similarity (as determined by their 
comparison score, not similarity score), and is used later in this work to extract potential lookalikes in our dataset for further analysis by the proposed similarity network. For the remaining comparison experiments, the approach presented in Howard et al. [39] was used, wherein all-toall matching was performed on each of the face datasets retaining only the non-mated, or impostor, distributions. The remaining comparison experiments correspond to each of the face datasets used in this work and are as follows: twin dataset, non-twin dataset, and the combined non-twin and CelebA dataset (referred to as the large scale non-twin dataset henceforth).

These match experiments are designed to show the effect of highly similar face pairs on two typical facial recognition tools, as well as identify potential look-alike pairs from the datasets used in this study. By first performing both mated and non-mated matching using only the identical twin pairs, a baseline of the worst case of facial recognition performance can be ascertained. As stated above, the average result of the non-mated comparison score distribution from this initial baseline experiment represents an experimental threshold for later matching experiments. This threshold represents the average non-mated comparison score of the absolute worst case presented to a face recognition tool, and it is expected that any non-mated comparison score from a non-twin pair falling above this threshold may be a look-alike pair. By performing all-to-all non-mated matching on all the datasets and extracting the pairs with comparison scores falling above the experimental threshold, these potential look-alike pairs can be extracted and examined further with the proposed similarity network described in the next section.

\subsection{Similarity Network}

To determine a quantitative measure of similarity between identical twins, a deep convolutional neural network was implemented. This network was designed with a Siamese 
architecture to directly compare two faces. Each half of this network was comprised of a FaceNet architecture, with the weights of the network shared between the two halves (Figure 14).

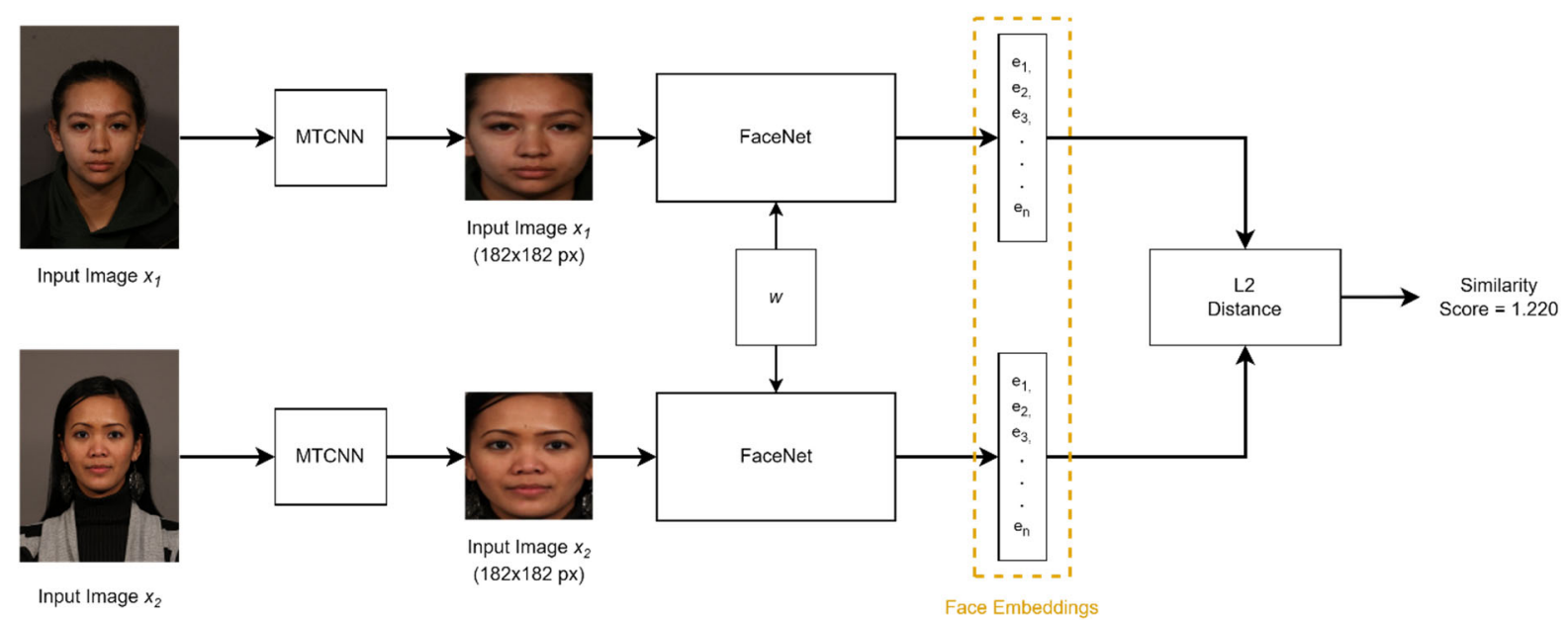

Figure 14: Similarity network diagram.

The FaceNet architecture was used as the foundation of this network for its high accuracy on typically difficult, similar face images. In addition to the high accuracy of the network, this network was also chosen for its ability to generate highly representative face embeddings. In the original publication [26], it is stated that, "The network is trained such that the squared L2 distances in the embedding space directly correspond to face similarity: faces of the same person have small distances and faces of distinct people have large distances." This is advantageous, as this work seeks to quantify the similarity of identical twin pairs.

The proposed network was optimized using the contrastive loss function shown in the equation below [40]:

$$
L=(1-y) D\left(x_{1}, x_{2}\right)^{2}+y * \max \left(0, m-D\left(x_{1}, x_{2}\right)\right)^{2}
$$

Equation 2: Contrastive loss equation. 
where $\left(x_{1}\right.$ and $\left.x_{2}\right)$ are the two images, $D(*)$ is the L2 difference between the embeddings generated by the network, $y$ is the ground-truth label for the image pair, and $m$ is the contrastive margin. Contrastive loss seeks to minimize the L2 distance between similar samples who reside close to one another in the feature space while maximizing the L2 distance between the dissimilar samples.

The output of this network consists of the L2 distance between two samples in the feature space. As this result gives similar samples a low score and dissimilar samples a high score, for clarity, the scores are inverted such that similar samples have a high score and dissimilar samples a low score. This was achieved by subtracting each resultant similarity score from the maximum similarity score in a given set of scores.

The training phase of the network for the task at hand consisted of fine tuning the weights with data from the twin dataset. Starting with network pre-trained on the VGGFace2 dataset, the network was fine-tuned on a subset of the twin database. This fine tuning consisted of training over four epochs at a learning rate of $1 \mathrm{e} 10^{-5}$ with a margin equal to 0.5 . This fine tuning was performed on a tailored verification task where a pair of identical twin images represents the positive case, and a pair of unrelated look-alikes represents the negative case. Training was stopped when the AUC of the network began to degrade on the verification task. This training encouraged the network to group together those samples with the facial similarity of identical twins in the embedded feature space, and inversely pushed apart those samples not as similar as identical twins.

The main training and testing dataset for this network was comprised of a subset of the twin dataset. This dataset contained images of identical twin pairs and imposter look-alikes to the twin pairs sorted into an equal number of genuine and impostor pairs, where a genuine pair consists of (Twin A vs. Twin B), and an impostor pair consists of (Twin A vs. look-alike). The look-alikes 
for each identity were found by selecting the identities with the highest FaceNet match score to each twin identity. This training schema is chosen because the network should learn to determine facial similarity from the most similar face pairs available (i.e., identical twins). It is expected that an individual's identical twin will be more similar to that individual than any potential look-alike, as such, the network is trained to identify the face pairs with the highest facial similarity. The dataset contains 645 identical twin identities, with a total of 3,203 images, split 80/20 for training and testing, respectively. The twin identities used in the training of the similarity network were chosen based upon their non-mated comparison scores from the FaceNet matcher against their identical twin. Approximately 50\% of the twin identities with the highest non-mated Twin A vs Twin B non-mated comparison scores make up the 645 training identities.

For both the training and testing of the network the input face images were sorted into dataset folders containing either A) images of twin A and twin B from an identical twin pair or B) images of twin A and images of twin A's look-alike(s) as described above. During training and testing the network was fed samples randomly from either folder A or B for each identity with a 50/50 chance of the input samples coming from either category A or B. This balances the input sample distribution so that on average the network learns from an equal number of positive and negative cases from the compiled dataset. 


\title{
Chapter 4: Results
}

\subsection{Match Experiment Results}

\author{
4.1.1 Identical Twin Baseline Experiments
}

Figure 15 and 16 illustrate the results of the identical twin baseline experiments, indicating that the average comparison score for identical twins trends higher than the comparison score for non-twin matches. The mean comparison score for identical twins in this baseline represents the experimental twin threshold $T$, and, when compared to the mated score distribution, this threshold approximates the left tail of the mated distribution for both matchers tested. This result indicates that for both matchers the mean non-mated comparison score for identical twins is a good estimator of the hardest cases presented to these matchers. 


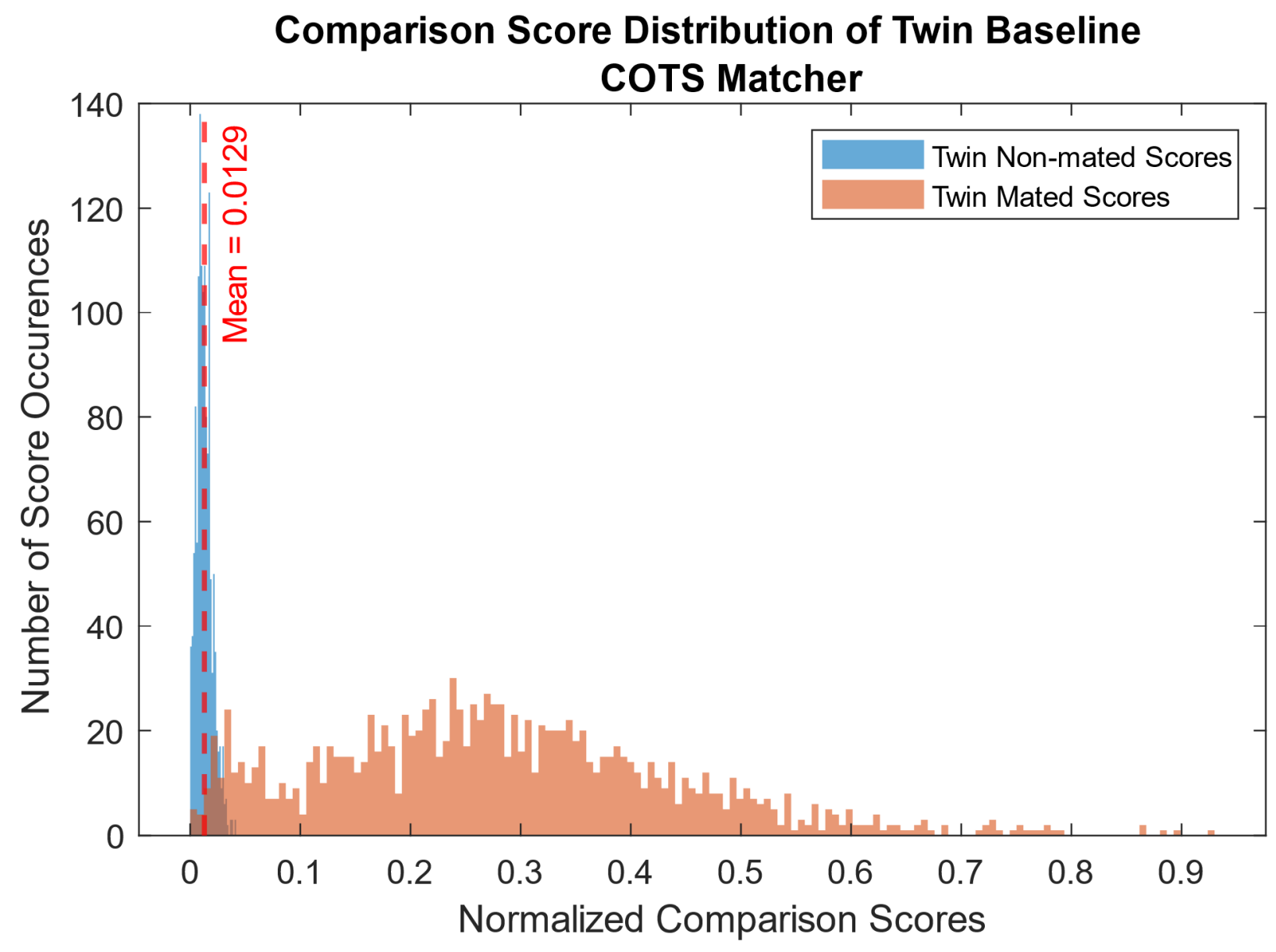

Figure 15: Twin baseline match experiment results, Neurotechnology matcher. 


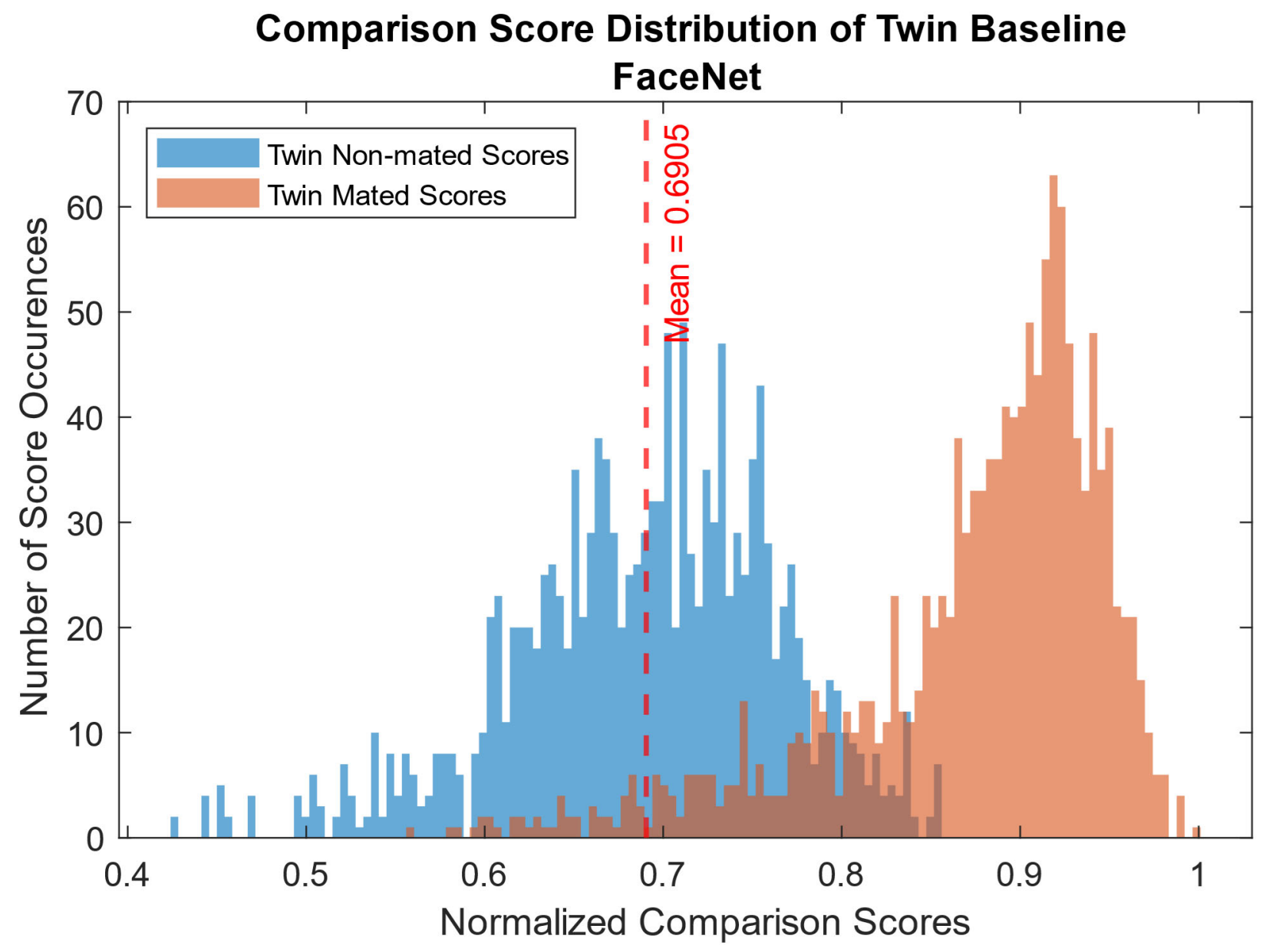

Figure 16: Twin baseline match experiment results, FaceNet matcher.

\subsubsection{All-to-all Non-mated Matching Experiments}

After the baseline matching experiments were performed, all-to-all impostor or non-mated matching was performed using both matchers. The comparison scores for each of these experiments were analyzed to extract the scores falling at and above the experimental twin threshold, $T$. 


\subsubsection{Twin Dataset Match Experiment Results}

The following plots (Figures 17 and 18) show the non-mated or impostor distributions from the twin dataset matching experiment. In this experiment "all-to-all" matching is performed using every identity in the dataset. The distributions for this experiment show that, as expected, the overwhelming majority of match scores fall below the twin threshold for both matchers. Additionally, most of the scores falling above the threshold are comparisons between two identical twins or two family members for both matchers tested.

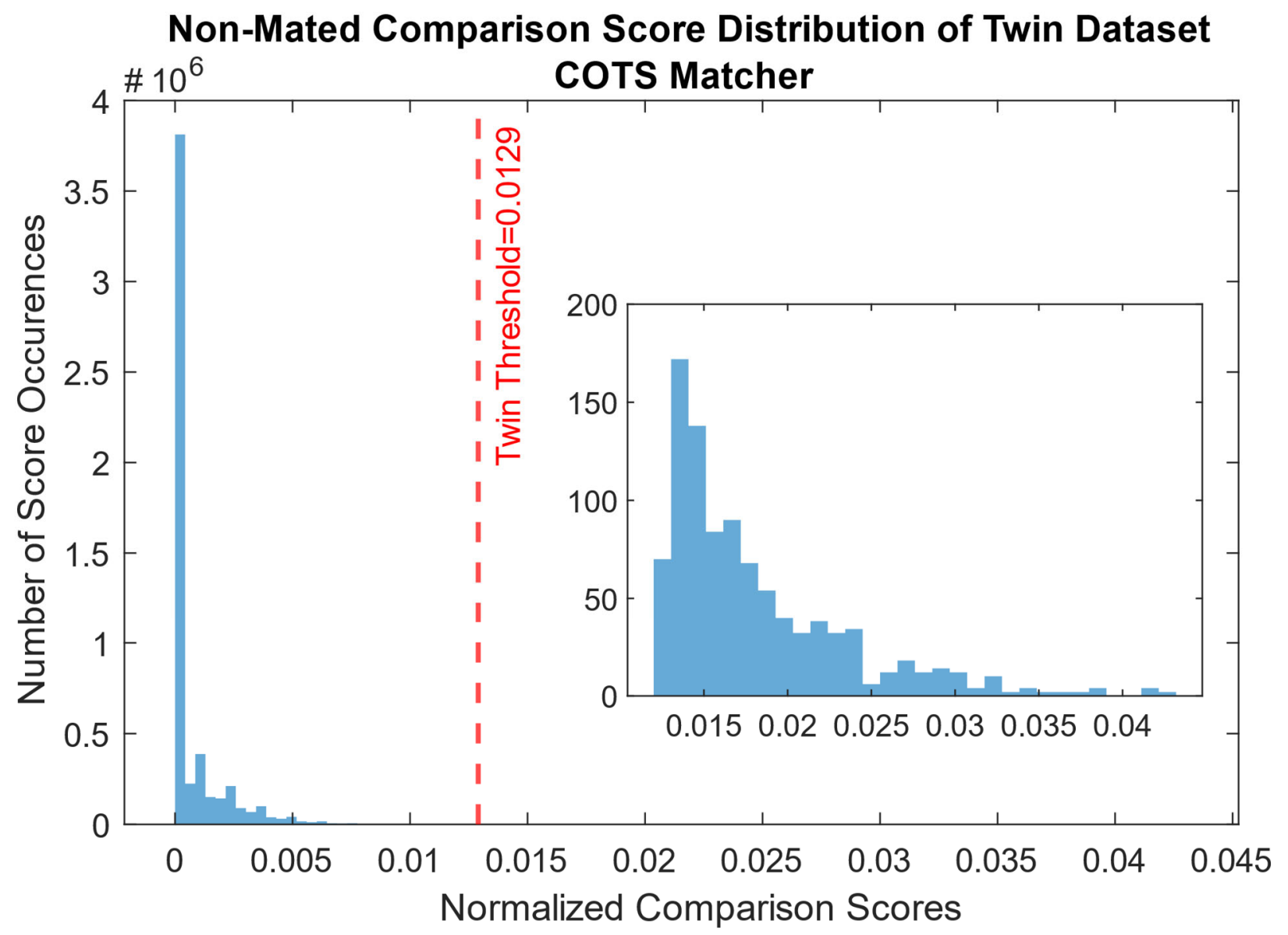

Figure 17: Twin dataset all-to-all non-mated match experiment results, Neurotechnology COTS matcher. 


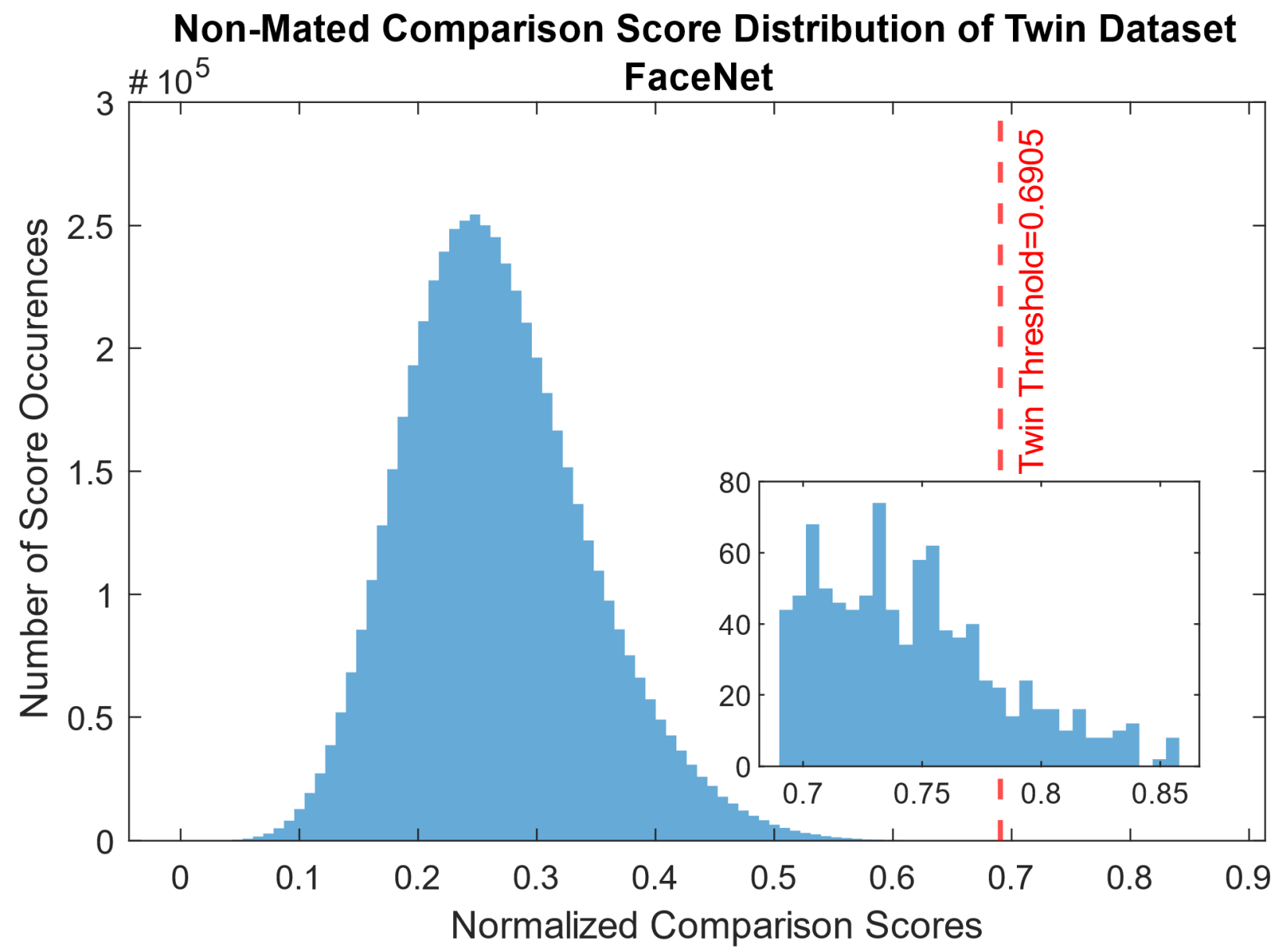

Figure 18: Twin dataset all-to-all non-mated match experiment results, FaceNet matcher.

\subsubsection{Non-twin Dataset Match Experiment Results}

For the non-twin dataset, as in the twin dataset experiment, all-to-all non-mated or impostor matching was performed. Similarly, the non-twin dataset experiment shows a large majority of the scores falling below the experimental threshold, as illustrated in Figures 19 and 20. As the identities in the non-twin datasets are unrelated individuals, the scores above the threshold represent potential look-alike pairs. 


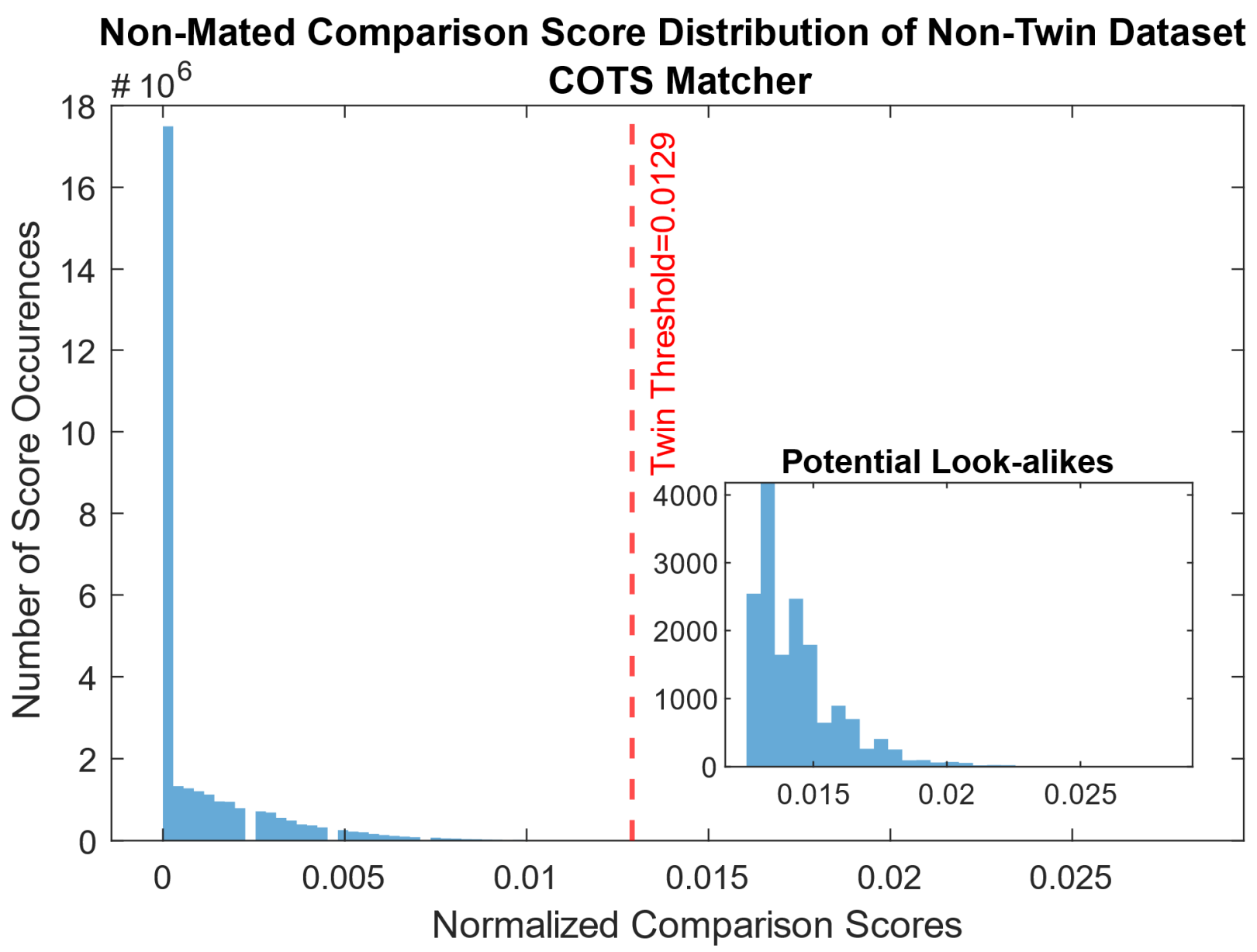

Figure 19: Non-twin dataset all-to-all non-mated match experiment results, Neurotechnology COTS matcher. 


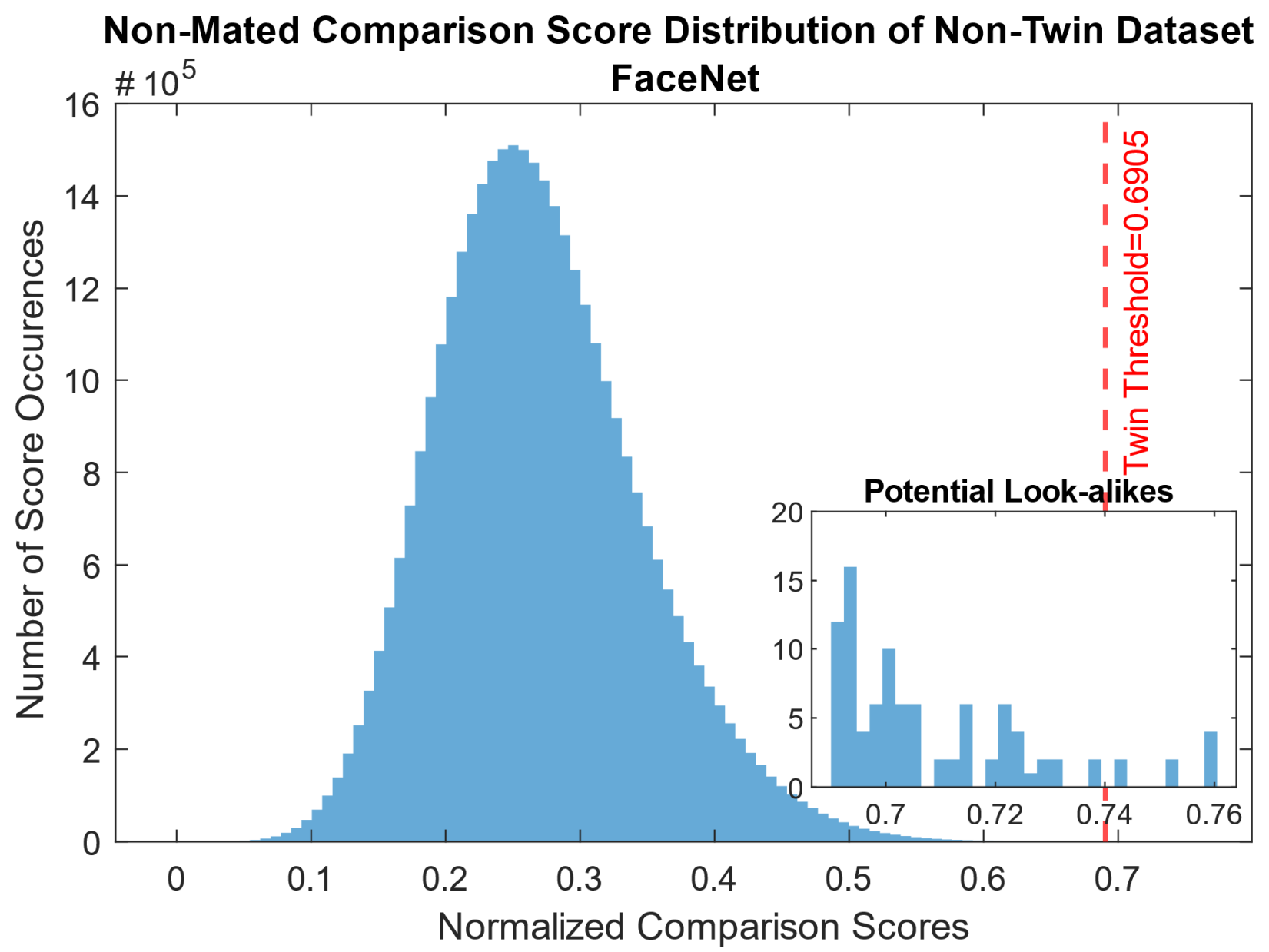

Figure 20: Non-twin dataset all-to-all non-mated match experiment results, FaceNet matcher.

\subsubsection{Large Scale Non-Twin Dataset Match Experiment Results}

Due to performance limitations of the COTS matcher, the final experiment was carried out only using the FaceNet matcher, with results presented in Figure 21. Again, in the large-scale nontwin experiment, the large majority of match scores fall below the twin threshold, but the scores above the threshold represent potential look-alike pairs. 
Non-Mated Comparison Score Distribution of Large Scale Non-Twin Dataset

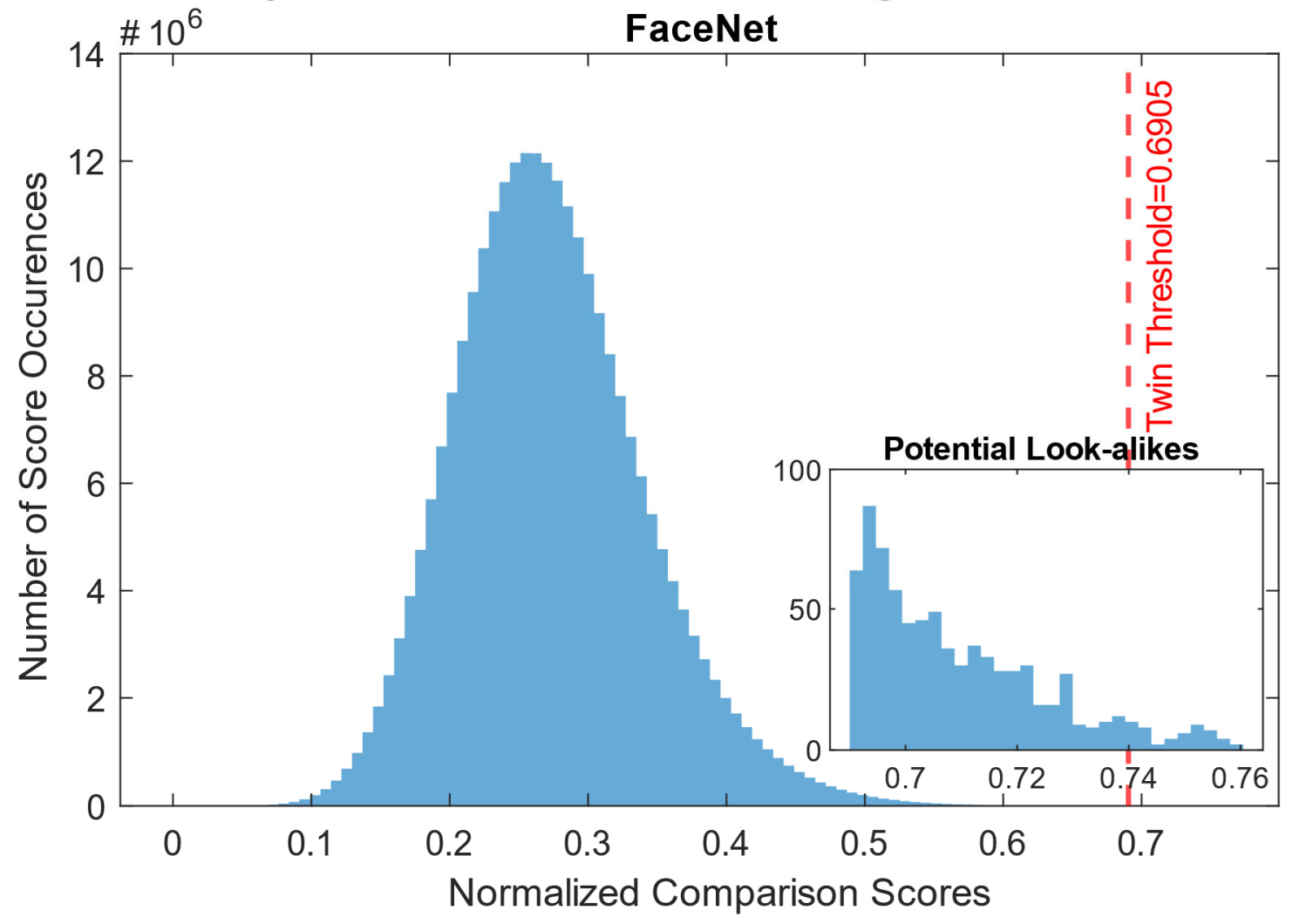

Figure 21: Large Scale non-twin dataset all-to-all non-mated match experiment results, FaceNet matcher.

\subsubsection{Match Scores Above Experimental Threshold $T$}

For each of the previous match experiments, the matches with scores above the chosen threshold were extracted for further analysis. Tables 2, 3, and 4 present the information about match scores falling above the twins threshold for each matcher and each dataset. 
Table 2: Twin dataset non-mated match experiment results, comparison scores above experimental twin threshold $\mathrm{T}$.

\begin{tabular}{|c|c|c|c|c|}
\hline \multicolumn{5}{|c|}{ Twin Dataset COTS Matcher } \\
\hline Relationship & $\begin{array}{l}\text { \# Scores } \\
>=T\end{array}$ & Average Score & Score Range & Percent of Matches \\
\hline Ident. Twin & 601 & 0.01865 & {$[0.0129-0.0431]$} & $0.0117 \%$ \\
\hline Ident. Mirror Twin & 112 & 0.02 & {$[0.0132-0.955]$} & $0.0022 \%$ \\
\hline Fraternal Twin & 48 & 0.0199 & {$[0.0135-0.799]$} & $0.00093 \%$ \\
\hline Mother & 1 & 0.0158 & {$[0.0158]$} & $0.000019 \%$ \\
\hline Child & 1 & 0.0158 & {$[0.0158]$} & $0.000019 \%$ \\
\hline No Relation & 199 & 0.0137 & [0.0129-0.0189] & $0.0038 \%$ \\
\hline Total & 962 & & & $0.0187 \%$ \\
\hline \multicolumn{5}{|c|}{ Twin Dataset FaceNet Matcher } \\
\hline Relationship & $\begin{array}{l}\text { \# Scores } \\
>=T\end{array}$ & Average Score & Score Range & Percent of Matches \\
\hline Ident. Twin & 724 & 0.746 & {$[0.6905-0.856]$} & $0.014 \%$ \\
\hline Ident. Mirror Twin & 144 & 0.746 & {$[0.6905-0.84]$} & $0.00279 \%$ \\
\hline Fraternal Twin & 50 & 0.753 & {$[0.6905-0.83]$} & $0.00097 \%$ \\
\hline No Relation & 4 & 0.706 & {$[0.694-0.7177]$} & $0.000077 \%$ \\
\hline Unknown & 2 & 0.694 & {$[0.694]$} & $0.000038 \%$ \\
\hline Total & 924 & & & $0.018 \%$ \\
\hline
\end{tabular}


Table 3: Non-twin dataset non-mated match experiment results, comparison scores above experimental twin threshold $\mathrm{T}$.

\begin{tabular}{|l|l|l|l|l|}
\hline \multicolumn{2}{|l|}{ Non-twin dataset - COTS Matcher } & Score Range & Percent of Matches \\
\hline Relationship & $\begin{array}{l}\text { \# Scores } \\
>=T\end{array}$ & Average Score & {$[0.0129-0.0283]$} & $0.0580 \%$ \\
\hline No Relation & 16274 & 0.0144 & \multicolumn{2}{|l|}{} \\
\hline \hline Non-twin dataset - FaceNet Matcher & Score Range & Percent of Matches \\
\hline Relationship & $\begin{array}{l}\text { \# Scores } \\
>=T\end{array}$ & Average Score & {$[0.6905-0.76]$} & $0.000346 \%$ \\
\hline No Relation & 97 & 0.704 & & \\
\hline
\end{tabular}

Table 4: Large scale non-twin dataset non-mated match experiment results, comparison scores above experimental twin threshold $\mathrm{T}$.

\begin{tabular}{|l|l|l|l|l|}
\hline \multicolumn{5}{|l|}{ Large Scale Non-twin dataset - FaceNet Matcher } \\
\hline Relationship & $\begin{array}{l}\text { \# Scores } \\
>=T\end{array}$ & Average Score & Score Range & Percent of Matches \\
\hline No Relation & 792 & 0.71 & {$[0.6905-0.76]$} & $0.000331 \%$ \\
\hline
\end{tabular}

In each of the matching experiments, it is shown that an overwhelming majority of match scores fall below the experimental twin threshold. This leads to the observation that impostor lookalikes are a rare occurrence in the population used in this study. Due to the relatively small number of identities in the datasets used for this evaluation, it is not possible to accurately predict the frequency of look-alike occurrence in the general population based on these results. However, the similarity measure described in the next section provides a method of finding highly similar faces in any given dataset. 


\subsection{Similarity Network Results}

\subsubsection{Network Training and Testing}

After training and testing, the proposed network achieved a train AUC of 0.917, and test AUC of 0.979 in the classification of a pair of face images as a twin pair or look-alike pair (Figures $22 \& 23)$. While the end goal of this network is not verification, the accuracy of the network on the tailored verification task shows that the network can accurately identify highly similar face pairs.

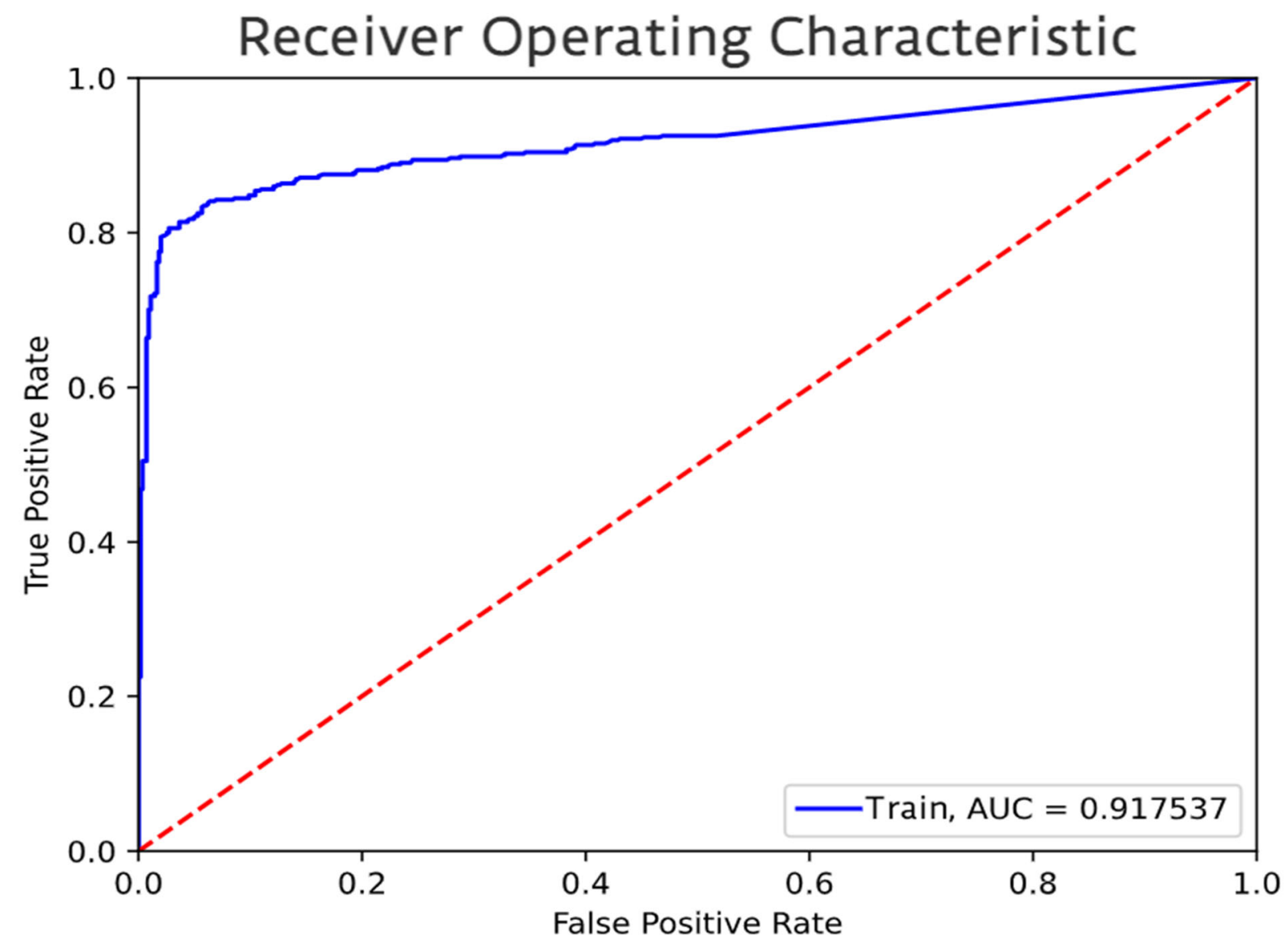

Figure 22: Train ROC curve of similarity network on the tailored verification task. 


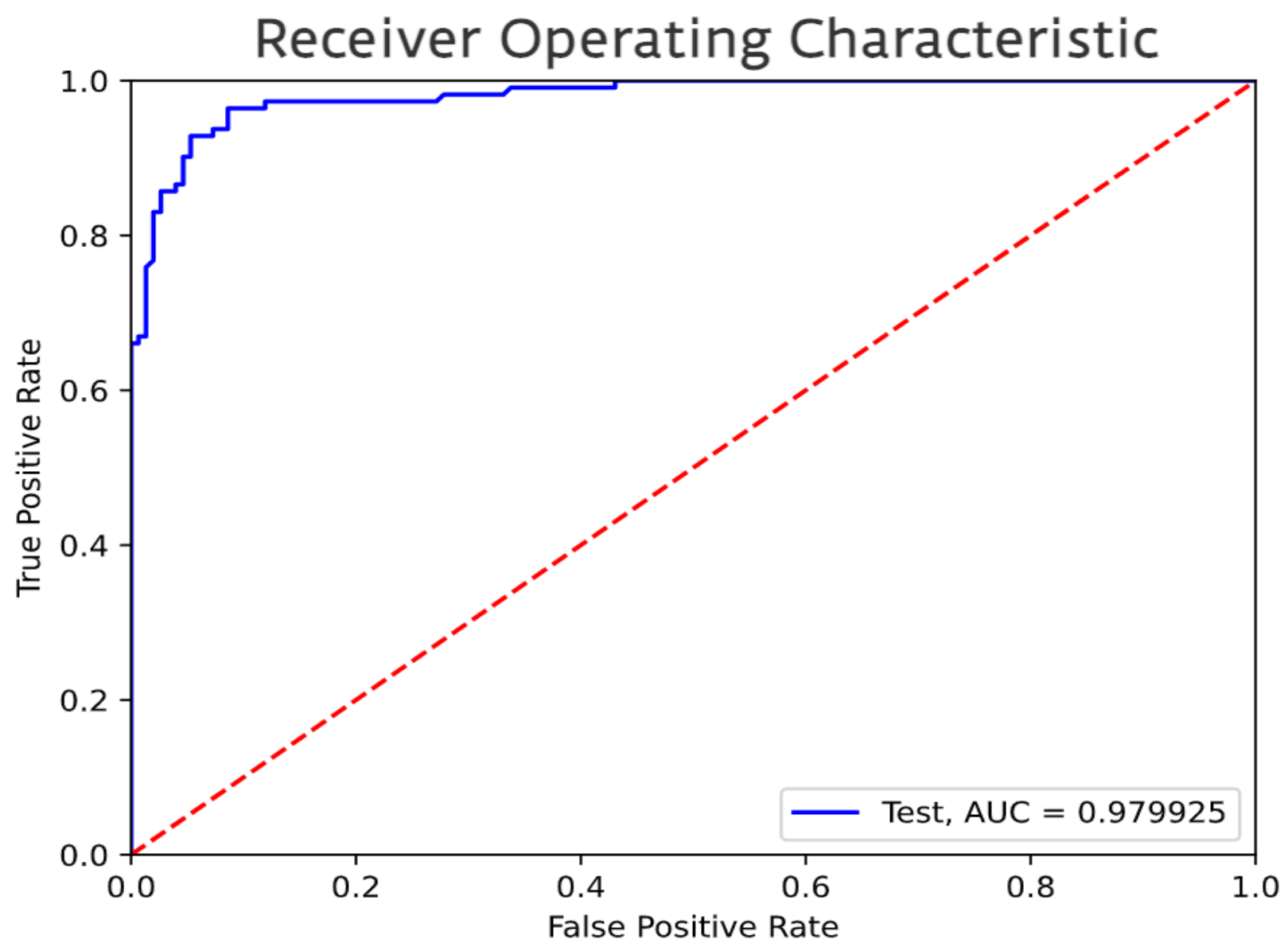

Figure 23: Test ROC curve of similarity network on the tailored verification task.

\subsubsection{Identical Twin Similarity Baseline}

This similarity network was then applied to both the twin dataset and large-scale non-twin dataset to observe the general similarity of twin and non-twin individuals. Initially, the similarity score of only the identical twin pairs was calculated (Figure 24). This distribution of similarity scores for identical twin pairs is the foundation of the worst-case baseline measure of similarity. As identical twins exist on a spectrum of similarity, two measurements of the baseline similarity between identical twin pairs are reported. The mean similarity score between identical twin pairs, 1.09 , captures the similarity of both highly similar and dissimilar twins, while the fourth quartile 
of the similarity score distribution, $\geq 1.29$, represents only the most similar twin pairs. In this experiment, the fourth quartile score of the distribution may more accurately represent the worst case of similarity presented to FR systems.

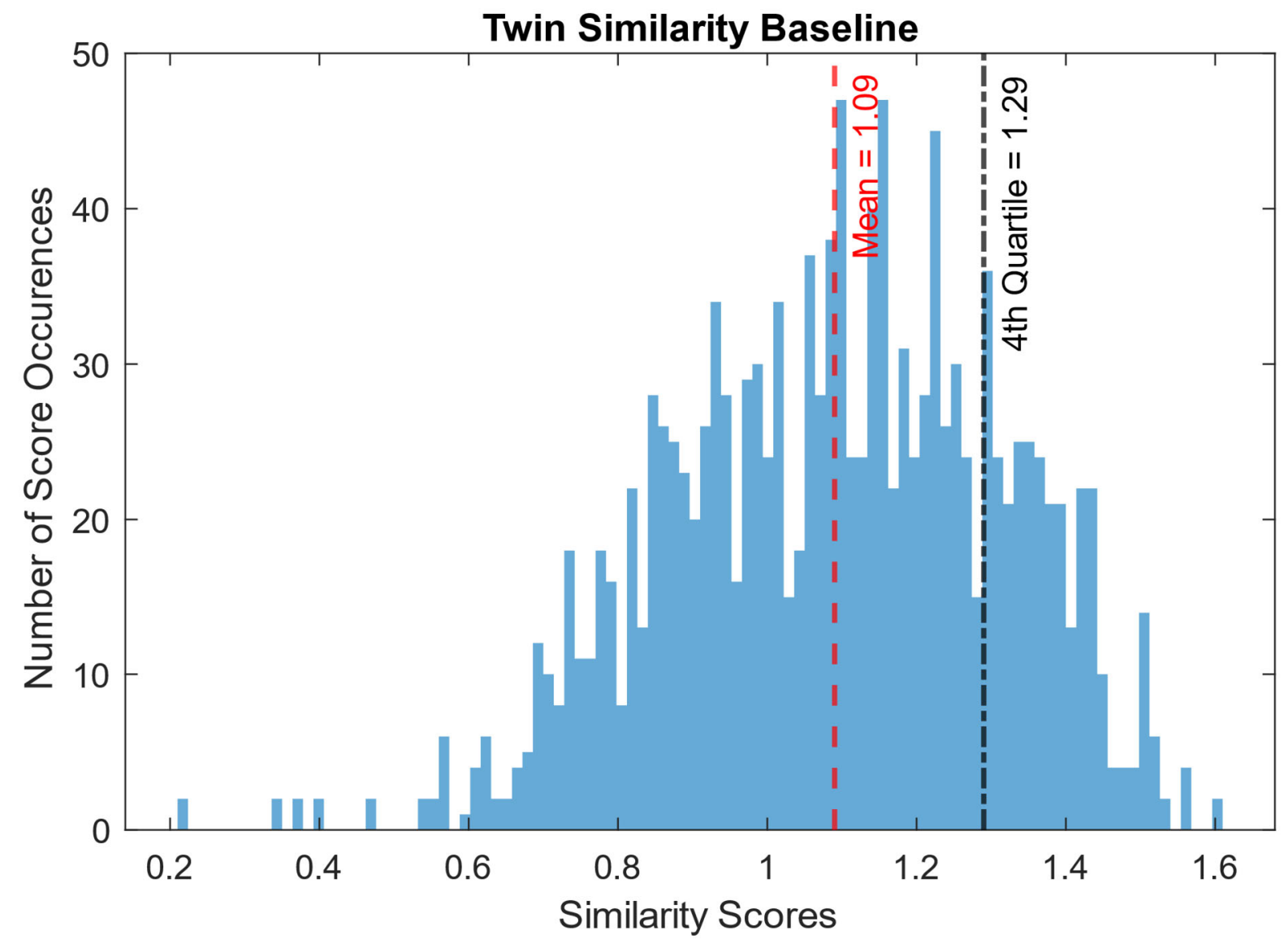

Figure 24: Twin similarity score baseline experiment.

\subsubsection{Look-alike Pair Extraction}

After determining the worst-case baseline for facial similarity, this measure was used to set the threshold for the similarity scores of the large-scale non-twin dataset. Since the network was fine-tuned using only ideal face images, the similarity score returned for "in-the-wild" face 
images may not be as robust as the similarity score returned for controlled images. Several examples of identical twin pairs and non-mated pairs with similarity scores exceeding the baseline measurements are shown in Figure 25.

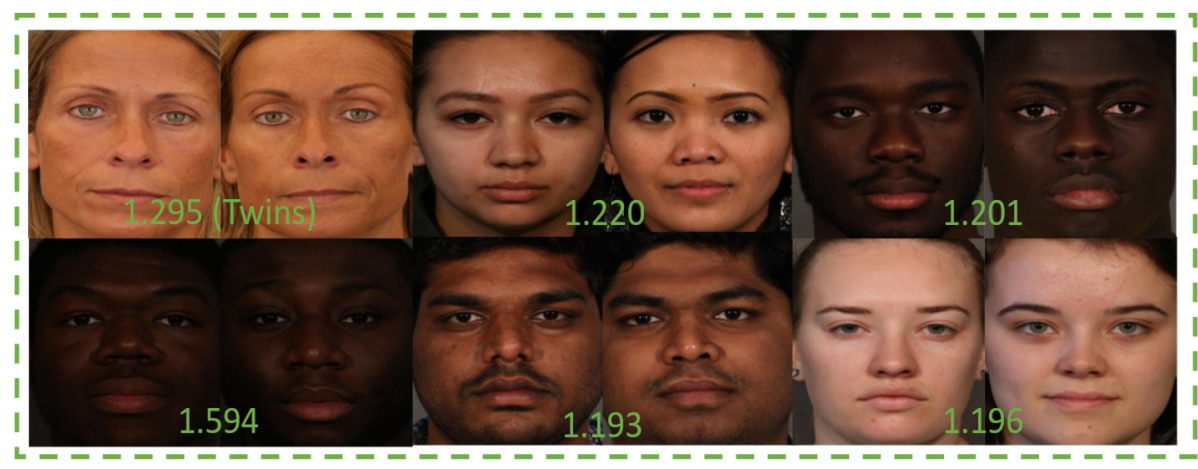

Highly Similar Identities

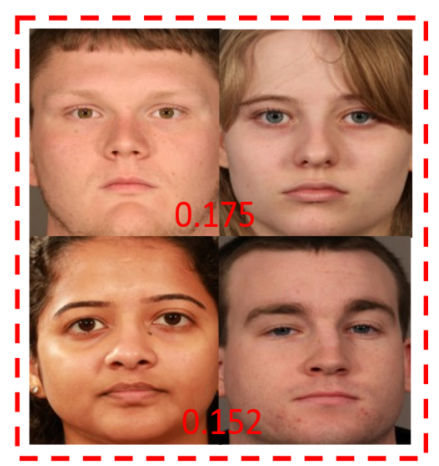

Dissimilar Identities

Figure 25: Examples of highly similar and dissimilar face pairs as determined by the proposed similarity network.

\subsubsection{Comparison Score Versus Similarity Score Analysis}

An additional analysis was performed to correlate the comparison score results of the COTS matcher to the similarity score obtained from our similarity network. Using the non-mated pairs whose comparison scores exceeded the experimental twin threshold in the matching experiments detailed above, a comparison was made to the similarity score calculated for the same pairs. Examples of individuals with high COTS comparison scores and the corresponding similarity score are highlighted below (see Figure 26). 


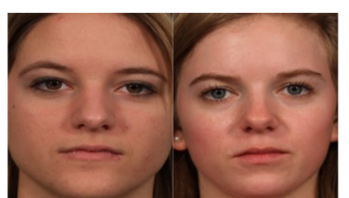

COTS Match Score $=0.649$

Similarity Score $=0.754$

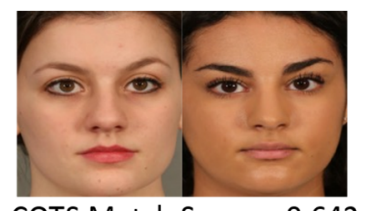

COTS Match Score $=0.642$ Similarity Score $=0.796$

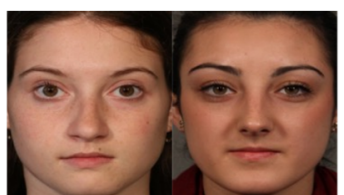

COTS Match Score $=0.567$ Similarity Score $=0.494$

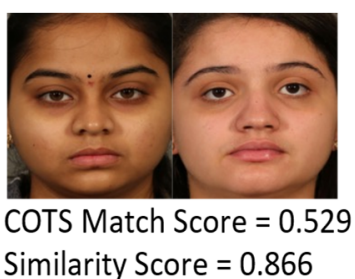

Similarity Score $=0.866$

Figure 26: High COTS match score face pairs and the corresponding similarity scores for each pair.

As Figure 26 indicates, the COTS comparison score for each of these face pairs was high; however, none of the pairs' similarity scores are above the twin similarity threshold. This indicates that the comparison score returned by the COTS matcher may not be directly correlated with facial similarity, and instead, may rely on other features of the image in its comparison score determination process.

A further analysis of the relationship between the comparison score returned by a facial recognition tool and the similarity score returned by the proposed network was carried out. The first experiment in this analysis shows a scatter plot on which each point represents the comparison score returned by the COTS matcher and the similarity score returned by the proposed network for each face comparison in the non-twin dataset (Figure 27). This same experiment was carried out for the FaceNet comparison scores and similarity scores returned for the large scale non-twin dataset (Figure 28). The trend line shown on each plot was determined via a linear polynomial curve fitting algorithm and shows the overall trend of the datapoints over the range of comparison scores. 


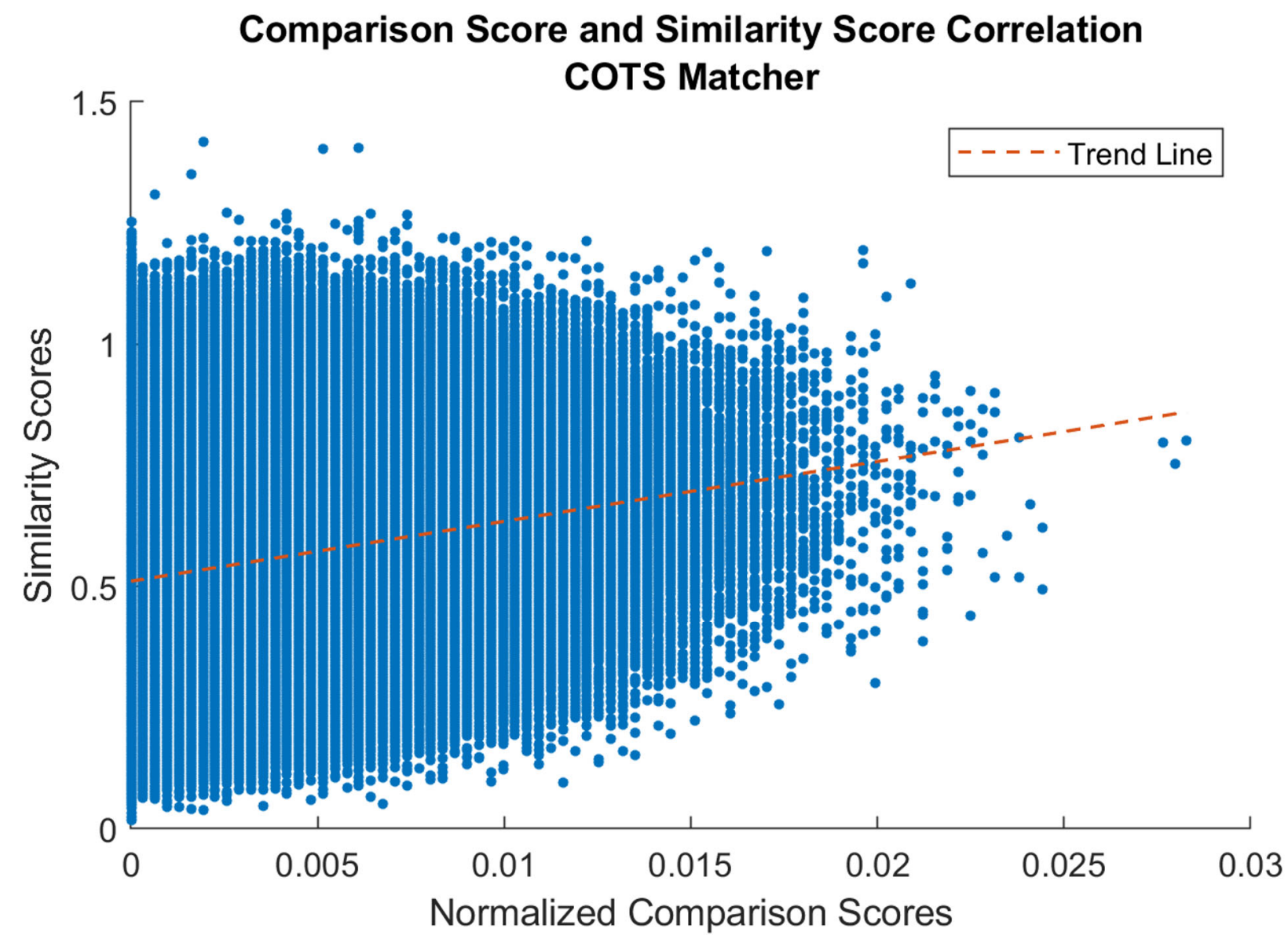

Figure 27: Correlation scatter plot of comparison scores and similarity scores, COTS matcher. 


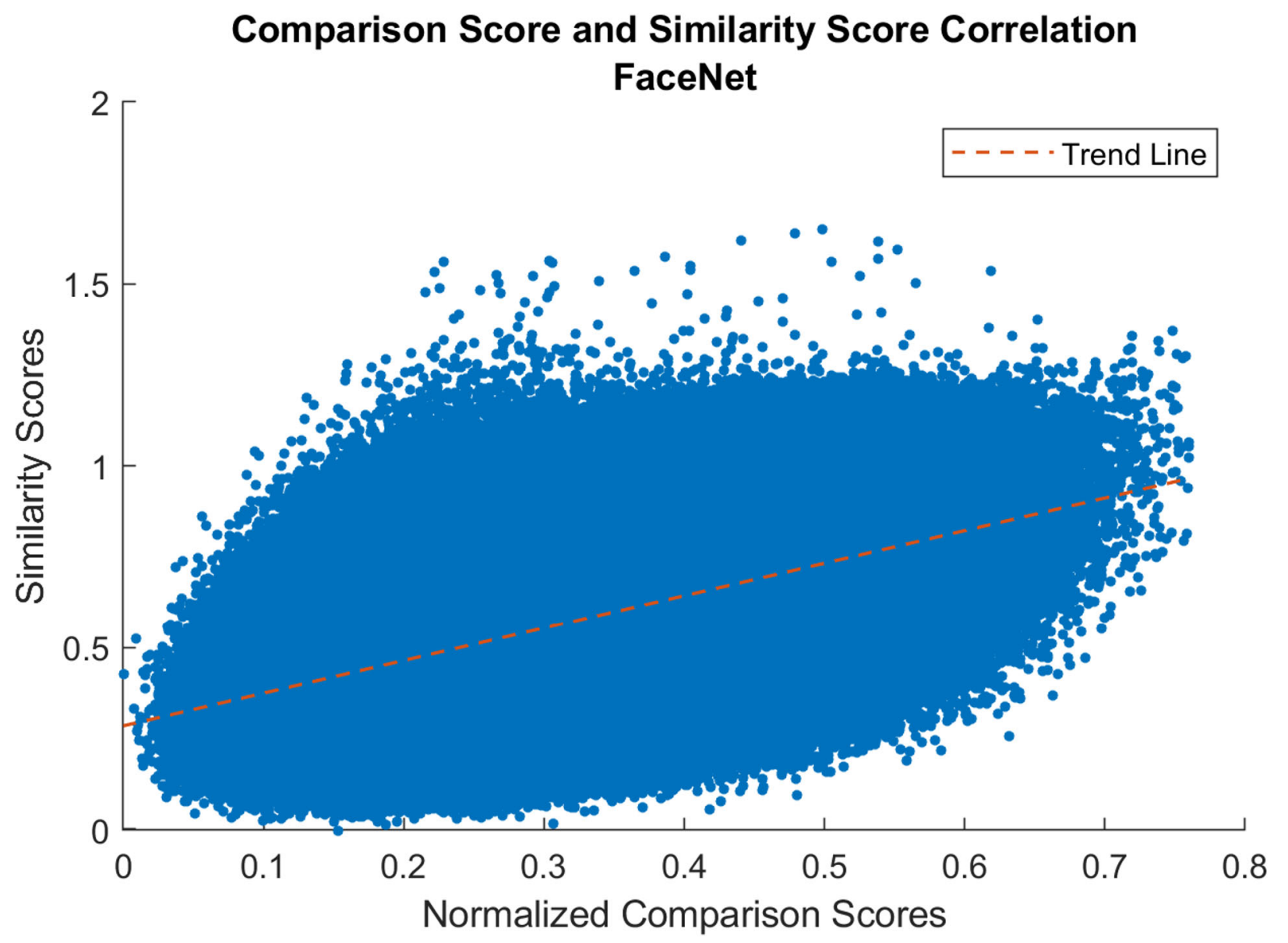

Figure 28: Correlation scatter plot of comparison scores and similarity scores, FaceNet matcher.

As shown in each of the above experiments, both the COTS matcher and the FaceNet matcher comparison scores show a positive trend with the similarity score returned by the proposed network for each of the tested face pairs. However, it is shown that for both tested matchers there is a wide range of similarity scores returned over the entire range of comparison scores for both matchers. These results indicate that while perceived facial similarity does play a role in the determination of the comparison score between face pairs, the facial similarity of the pair may not be the chief factor in the determination of comparison score. This is the expected result, as facial recognition is a distinct challenge from that of determining facial similarity, and the comparison 
score returned by a facial recognition tool is generally tuned to maximize recognition performance. This is especially true for the COTS matcher used in this work, as this matcher utilizes face template comparisons which may focus on features that do not correspond directly to perceived facial similarity. This is also true for the FaceNet matcher, because while the authors claim that the network's face embeddings are directly correlated with facial similarity, the network is optimized for maximal facial recognition performance. This may lead the network to produce embeddings which are designed for facial recognition comparisons, and not the determination of facial similarity.

\subsubsection{Frequency of Look-alike Identity Occurrence Estimation}

Finally, an investigation into the number of potential look-alike pairs returned by the network while varying the similarity threshold was performed to further understand the occurrence of look-alikes in a given population of unrelated individuals (Figure 29). 


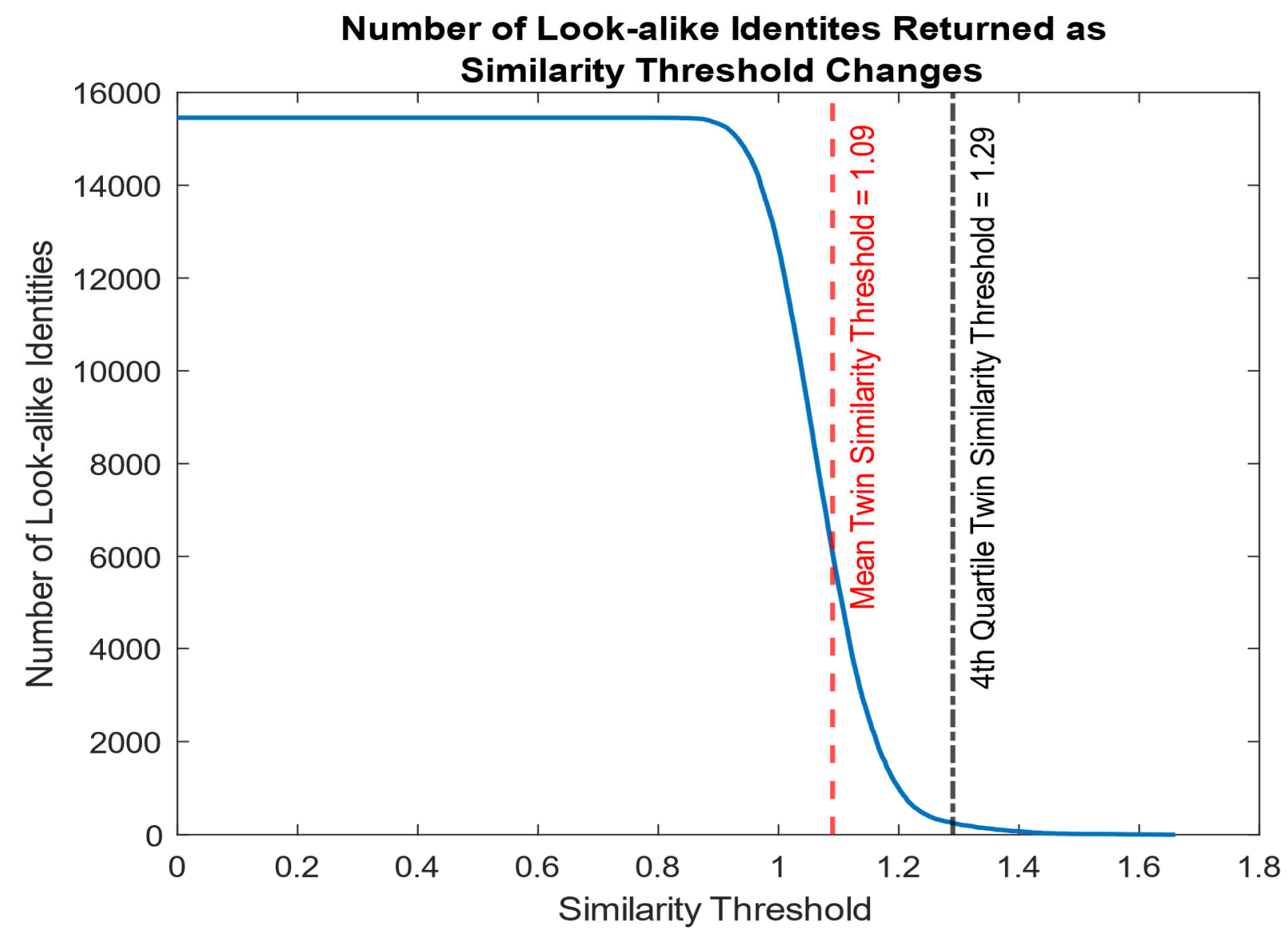

Figure 29: Number of look-alike identities returned from the large scale non-twin dataset based on similarity threshold used.

Given the mean twin similarity threshold of $1.09,6,153$ of the total 15,455 identities in the large-scale non-twin dataset have at least one similarity comparison at or above the threshold. This means $39.8 \%$ of the identities have one or more potential look-alike at this level of similarity. At and above the fourth quartile threshold, only 228 identities have one or more potential look-alike, or $1.475 \%$ of identities in the dataset. While there are not enough identities present in the datasets used in this work to estimate the frequency of look-alike occurrence in general using the proposed network, given a large enough dataset such an analysis could be carried out. Furthermore, the 
proposed network can be applied to any dataset to gauge the number of look-alike identities within the dataset by applying the worst-case similarity threshold to an all-to-all non-mated similarity comparison for the dataset. 


\section{Chapter 5: Conclusions}

\subsection{Conclusion}

This work explores the problem of facial similarity and the facial recognition of identical twins and look-alike or doppelganger pairs. These face pairs represent the hardest cases presented to facial recognition systems and examining the facial similarity of these pairs allows for further exploration into the relationship between facial similarity and the comparison score returned by a facial recognition system. The primary goal of this work is to quantify the facial similarity of identical twins in general. This similarity measurement represents the worst-case baseline of facial similarity in any non-mated comparison in facial recognition. This measure was developed via a deep convolutional neural network, trained specifically to identify highly similar face pairs. This network returns a quantitative measure of facial similarity (i.e., a similarity score) for any two faces, and has many applications including identifying look-alike pairs from large scale face datasets. Additionally, match experimentation was carried out to demonstrate the effect of highly similar faces on both commercial off the shelf and academic machine learning based facial recognition tools and identify potential look-alike identities. Using an experimental threshold determined from the mean identical twin non-mated match score, potential look-alike pairs were extracted from the populations used in this study through match experimentation.

Several conclusions can be drawn from the results of this work. First, look-alike pairs are quite rare in the populations contained within the datasets used for this study. This is confirmed by both the match experiments carried out using both a commercial off the shelf matcher, an academic machine learning based matcher, and the proposed similarity network. Due to the relatively low frequency of look-alikes contained within the data sets used, an accurate estimate 
of the frequency of look-alike pairs in general was not carried out, however the results of the proposed similarity network show that the network can identify similar face pairs from any face dataset. This result has applications in several spheres including: finding suitably similar face for morph face generation, determining the difficulty of any given dataset by the number of look-alike identities contained within, and further investigation into the relationship between comparison score and perceived similarity of any two faces. Second, the result show that the comparison score returned by a commercial off the shelf facial recognition tool are not directly correlated with the perceived visual similarity determined by the proposed network. This indicates that the commercial off the shelf facial recognition tool and the neural network-based tool used in the match experimentation in this work may not determine the comparison score between two faces directly from a measure of facial similarity, and instead determines it comparison scores via some feature set designed to maximize facial recognition performance. Finally, the difficulty that both identical twin pairs and look-alike pairs pose to facial recognition systems has again been demonstrated, as it has in previous works. This conclusion is drawn from the initial match experimentation carried out on identical twin pairs, and the high comparison scores returned from non-twin look-alike pairs falling above the experimental threshold determined via the identical twin match experimentation.

\subsection{Future Work}

There are several topics of interest for future studies in this area. The first is an adaptation of the birthday paradox to the occurrence of look-alikes in a population. Much like the birthday paradox seeks to calculate the probability of two people in a population sharing a birthday, calculating the probability of two unrelated individuals having high facial similarity based on the 
number of identities in a dataset would be a useful measure as face datasets continue to grow. This network would allow for the determination of such a measurement. The second is an application of the proposed similarity network in the generation of morphed faces. As the proposed network can identify similar faces from any dataset, using these look-alike pairs as suitably similar faces for morphed face pairs could further confirm the accuracy of the determination of facial similarity by the similarity network. Finally, the proposed network could be used to further investigate the role that facial similarity plays in the determination of comparison score by popular facial recognition tools. As shown in preliminary results in this work correlating the comparison score returned by two facial recognition tools and the similarity score returned by the proposed network, the comparison score returned by the facial recognition tools does not seem to be directly correlated with the similarity score returned by the proposed network. This experimentation could be extended to other commercial off the shelf facial recognition tools, or even machine learning based facial recognition tools, to better understand the role that facial similarity plays in the determination of a comparison score between two faces. 


\section{References}

[1] J. R. Paone et al., "Double Trouble: Differentiating Identical Twins by Face Recognition," IEEE Transactions on Information Forensics and Security, vol. 9, no. 2, pp. 285-295, Feb. 2014, doi: 10.1109/TIFS.2013.2296373.

[2] X. Sun, A. Torfi, and N. Nasrabadi, "Deep Siamese Convolutional Neural Networks for Identical Twins and look-alike Identification," in Deep Learning in Biometrics, CRC Press, 2018.

[3] A. K. Jain, S. Prabhakar, and S. Pankanti, "On the similarity of identical twin fingerprints," Pattern Recognition, vol. 35, pp. 2653-2663, 2002.

[4] Z. Sun, A. A. Paulino, J. Feng, Z. Chai, T. Tan, and A. K. Jain, "A study of multibiometric traits of identical twins," in Biometric Technology for Human Identification VII, Apr. 2010, vol. 7667, p. 76670T. doi: 10.1117/12.851369.

[5] H. Nejati, L. Zhang, T. Sim, E. Martinez-Marroquin, and G. Dong, "Wonder ears: Identification of identical twins from ear images," in Proceedings of the 21st International Conference on Pattern Recognition (ICPR2012), Nov. 2012, pp. 1201-1204.

[6] K. Ricanek and G. Mahalingam, "Biometrically, How Identical Are Identical Twins?," Computer, vol. 46, no. 3, pp. 94-96, Mar. 2013, doi: 10.1109/MC.2013.82.

[7] K. W. Bowyer and P. J. Flynn, "Biometric identification of identical twins: A survey," in 2016 IEEE 8th International Conference on Biometrics Theory, Applications and Systems (BTAS), Sep. 2016, pp. 1-8. doi: 10.1109/BTAS.2016.7791176.

[8] P. J. Phillips et al., "Distinguishing identical twins by face recognition," in 2011 IEEE International Conference on Automatic Face Gesture Recognition (FG), Mar. 2011, pp. 185-192. doi: 10.1109/FG.2011.5771395.

[9] M. T. Pruitt, J. M. Grant, J. R. Paone, P. J. Flynn, and R. W. V. Bruegge, "Facial recognition of identical twins," in 2011 International Joint Conference on Biometrics (IJCB), Oct. 2011, pp. 1-8. doi: 10.1109/IJCB.2011.6117476.

[10] B. Klare, A. A. Paulino, and A. K. Jain, "Analysis of facial features in identical twins," in 2011 International Joint Conference on Biometrics (IJCB), Oct. 2011, pp. 1-8. doi: 10.1109/IJCB.2011.6117548.

[11] G. Mahalingam and K. Ricanek, "Investigating the effects of gender and age group based differences in identical twins," in 2013 Fourth National Conference on Computer Vision, Pattern Recognition, Image Processing and Graphics (NCVPRIPG), Dec. 2013, pp. 1-4. doi: 10.1109/NCVPRIPG.2013.6776241.

[12] M. Kosmerlj, T. Fladsrud, E. Hjelmås, and E. Snekkenes, "Face Recognition Issues in a Border Control Environment," Jan. 2006, vol. 3832, pp. 33-39. doi: 10.1007/11608288_5.

[13] H. Lamba, A. Sarkar, M. Vatsa, R. Singh, and A. Noore, "Face recognition for look-alikes: A preliminary study," in 2011 International Joint Conference on Biometrics (IJCB), Oct. 2011, pp. 1-6. doi: 10.1109/IJCB.2011.6117520. 
[14] C. Rathgeb et al., "Impact of Doppelgängers on Face Recognition: Database and Evaluation," in 2021 International Conference of the Biometrics Special Interest Group (BIOSIG), Sep. 2021, pp. 1-4. doi: 10.1109/BIOSIG52210.2021.9548306.

[15] N. Ramanathan, R. Chellappa, and A. K. R. Chowdhury, "Facial similarity across age, disguise, illumination and pose," in 2004 International Conference on Image Processing, 2004. ICIP '04., Oct. 2004, vol. 3, pp. 1999-2002 Vol. 3. doi: 10.1109/ICIP.2004.1421474.

[16] A. Sadovnik, W. Gharbi, T. Vu, and A. Gallagher, "Finding your Lookalike: Measuring Face Similarity Rather than Face Identity," in 2018 IEEE/CVF Conference on Computer Vision and Pattern Recognition Workshops (CVPRW), Salt Lake City, UT, USA, Jun. 2018, pp. 2408-24088. doi: 10.1109/CVPRW.2018.00311.

[17] A. Röttcher, U. Scherhag, and C. Busch, "Finding the Suitable Doppelgänger for a Face Morphing Attack," in 2020 IEEE International Joint Conference on Biometrics (IJCB), Sep. 2020, pp. 1-7. doi: 10.1109/IJCB48548.2020.9304878.

[18] J. Deng, J. Guo, N. Xue, and S. Zafeiriou, "ArcFace: Additive Angular Margin Loss for Deep Face Recognition," in 2019 IEEE/CVF Conference on Computer Vision and Pattern Recognition (CVPR), Jun. 2019, pp. 4685-4694. doi: 10.1109/CVPR.2019.00482.

[19] G. B. Huang, M. Ramesh, T. Berg, and E. Learned-Miller, "Labeled Faces in the Wild: A Database for Studying Face Recognition in Unconstrained Environments," University of Massachusetts, Amherst, 2007.

[20] "Information technology — Vocabulary — Part 37: Biometrics," ISO/IEC 2382-37, 2017.

[21] A. K. Jain, A. A. Ross, and K. Nandakumar, Introduction to biometrics. New York: Springer, 2011.

[22] T. Kanade, "Picture Processing System by Computer Complex and Recognition of Human Faces.” Kyoto University (京都大学), May 23, 1974.

[23] M. Turk and A. Pentland, "Eigenfaces for Recognition," Journal of Cognitive Neuroscience, vol. 3, no. 1, pp. 71-86, Jan. 1991, doi: 10.1162/jocn.1991.3.1.71.

[24] P. N. Belhumeur, J. P. Hespanha, and D. J. Kriegman, "Eigenfaces vs. Fisherfaces: recognition using class specific linear projection," IEEE Transactions on Pattern Analysis and Machine Intelligence, vol. 19, no. 7, pp. 711-720, Jul. 1997, doi: 10.1109/34.598228.

[25] Y. Taigman, M. Yang, M. Ranzato, and L. Wolf, "DeepFace: Closing the Gap to HumanLevel Performance in Face Verification," in 2014 IEEE Conference on Computer Vision and Pattern Recognition, Jun. 2014, pp. 1701-1708. doi: 10.1109/CVPR.2014.220.

[26] F. Schroff, D. Kalenichenko, and J. Philbin, "FaceNet: A Unified Embedding for Face Recognition and Clustering," 2015 IEEE Conference on Computer Vision and Pattern Recognition (CVPR), pp. 815-823, Jun. 2015, doi: 10.1109/CVPR.2015.7298682.

[27] W. S. Mcculloch and W. Pitts, "A logical calculus of the ideas immanent in nervous activity," Bulletin of Mathematical Biophysics, vol. 5, pp. 115-133, 1943.

[28] D. O. Hebb, The organization of behavior: a neuropsychological theory. Mahwah, N.J: L. Erlbaum Associates, 2002.

[29] F. Rosenblatt, "The perceptron - A perceiving and recognizing automaton," Cornell Aeronautical Laboratory, Ithaca, New York, 85-460-1, Jan. 1957. 
[30] Y. LeCun, K. Kavukcuoglu, and C. Farabet, "Convolutional networks and applications in vision," in Proceedings of 2010 IEEE International Symposium on Circuits and Systems, May 2010, pp. 253-256. doi: 10.1109/ISCAS.2010.5537907.

[31] "Max-pooling / Pooling - Computer Science Wiki." https://computersciencewiki.org/index.php/Max-pooling_/Pooling.

[32] A. Krizhevsky, I. Sutskever, and G. E. Hinton, "ImageNet Classification with Deep Convolutional Neural Networks," in Advances in Neural Information Processing Systems, 2012, vol. 25.

[33] C. Szegedy et al., "Going deeper with convolutions," in 2015 IEEE Conference on Computer Vision and Pattern Recognition (CVPR), Boston, MA, USA, Jun. 2015, pp. 1-9. doi: 10.1109/CVPR.2015.7298594.

[34] O. Moindrot, "Triplet Loss and Online Triplet Mining in TensorFlow," Olivier Moindrot blog, Mar. 19, 2018. https://omoindrot.github.io/triplet-loss.

[35] National Institute of Standards and Technology, "American National Standard for Information Systems - Data Format for the Interchange of Fingerprint, Facial \& Other Biometric Information.” 2011.

[36] Z. Liu, P. Luo, X. Wang, and X. Tang, "Deep Learning Face Attributes in the Wild," in 2015 IEEE International Conference on Computer Vision (ICCV), Dec. 2015, pp. 37303738. doi: 10.1109/ICCV.2015.425.

[37] K. Zhang, Z. Zhang, Z. Li, and Y. Qiao, "Joint Face Detection and Alignment using Multitask Cascaded Convolutional Networks," IEEE Signal Process. Lett., vol. 23, no. 10, pp. 1499-1503, Oct. 2016, doi: 10.1109/LSP.2016.2603342.

[38] "VeriLook face identification technology, algorithm and SDK for PC, smartphones and Web." https://www.neurotechnology.com/verilook.html.

[39] J. J. Howard, Y. B. Sirotin, and A. R. Vemury, "The Effect of Broad and Specific Demographic Homogeneity on the Imposter Distributions and False Match Rates in Face Recognition Algorithm Performance," p. 8, 2019.

[40] R. Hadsell, S. Chopra, and Y. LeCun, "Dimensionality Reduction by Learning an Invariant Mapping," in 2006 IEEE Computer Society Conference on Computer Vision and Pattern Recognition (CVPR'06), Jun. 2006, vol. 2, pp. 1735-1742. doi: 10.1109/CVPR.2006.100. 